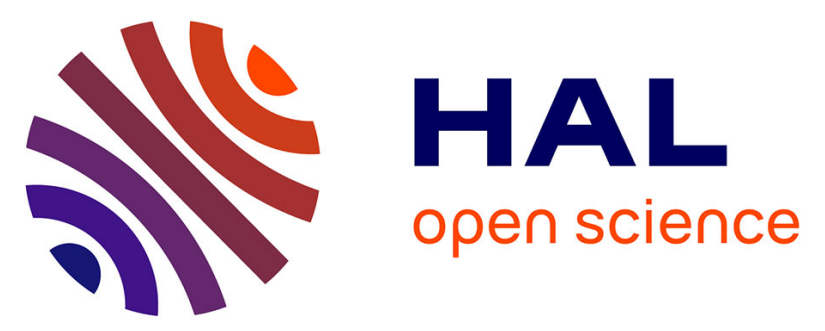

\title{
Michael Addition Initiated Substrate- or Catalyst-Controlled Chemodivergent Three-Component Construction of Fused and Bridged Polycyclic Heterocycles
}

Haiying Du, Jean Rodriguez, Xavier Bugaut, Thierry Constantieux

\section{To cite this version:}

Haiying Du, Jean Rodriguez, Xavier Bugaut, Thierry Constantieux. Michael Addition Initiated Substrate- or Catalyst-Controlled Chemodivergent Three-Component Construction of Fused and Bridged Polycyclic Heterocycles. Synthesis: Journal of Synthetic Organic Chemistry, 2015, 47 (15), pp.2188-2198. 10.1055/s-0034-1380229 . hal-01224292

\section{HAL Id: hal-01224292 \\ https://hal.science/hal-01224292}

Submitted on 30 May 2016

HAL is a multi-disciplinary open access archive for the deposit and dissemination of scientific research documents, whether they are published or not. The documents may come from teaching and research institutions in France or abroad, or from public or private research centers.
L'archive ouverte pluridisciplinaire HAL, est destinée au dépôt et à la diffusion de documents scientifiques de niveau recherche, publiés ou non, émanant des établissements d'enseignement et de recherche français ou étrangers, des laboratoires publics ou privés. 


\section{Michael Addition Initiated Substrate- or Catalyst-Controlled Chemodivergent Three-Component Construction of Fused and Bridged Polycyclic Heterocycles}

\author{
Haiying Du \\ Jean Rodriguez \\ Xavier Bugaut* \\ Thierry Constantieux* \\ Aix Marseille Université, Centrale Marseille, CNRS, \\ iSm2 UMR 7313, 13397, Marseille, France \\ xavier.bugaut@univ-amu.fr \\ thierry.constantieux@univ-amu.fr
}

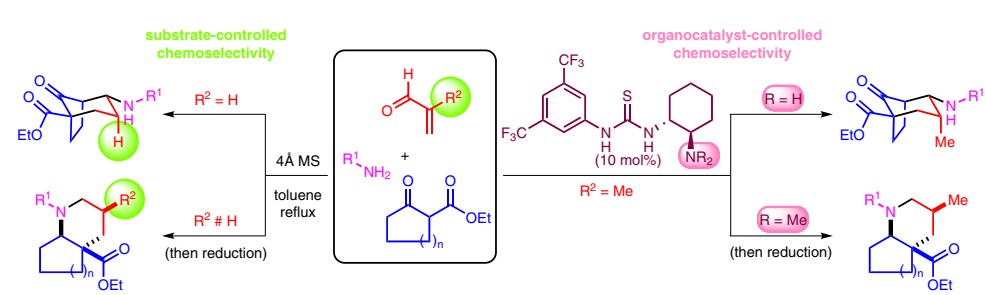

Received: 30.10 .2014

Accepted after revision: 23.01.2015

Published online: 11.03 .2015

DOI: 10.1055/s-0034-1380229; Art ID: ss-2014-z663-op

Abstract The results on the multicomponent reaction between cyclic $\beta$-keto esters, enals, and simple primary aliphatic amines are reported. In thermal conditions in the presence of $4 \AA$ molecular sieves, two different families of heterocyclic products (aminobicyclo[3.3.1]nonanones or cycloalka[b]piperidines) were obtained depending on the presence of a substituent in the $\alpha$-position of the aldehyde. Moreover, preliminary results on the possibility to obtain these two bicyclic cores from the very same starting materials by introducing organocatalysts with different activation modes in the reaction are presented.

Key words 1,3-dicarbonyls, heterocycles, Michael addition, multicomponent reaction, organocatalysis

Heteroatom-containing bridged and fused polycycles are ubiquitous motives that are present both in natural products and bioactive compounds, and new methods to synthesize them are continuously being invented. One particularly efficient strategy is the use of domino multicomponent reactions that consist in the chemoselective assembly of at least three organic molecules to afford structurally complex architectures. ${ }^{1,2}$ For more than ten years, our group has been involved in the development of new multicomponent reactions that make use of the Michael addition of 1,3-dicarbonyl compounds to trigger the formation of valuable products, including a large variety of heterocycles. ${ }^{3}$ In this context, we studied the combination of ethyl 1benzyl-4-oxopiperidine-3-carboxylate (1), acrolein, and primary amines in the presence of $4 \AA$ molecular sieves (MS) in refluxing toluene and observed a chemodivergent reaction, depending on the substitution of the amine (Scheme 1 , previous strategy): ${ }^{4} \alpha$-branched primary amines favored the formation of bridged aminoazabicyclo[3.3.1] nonanones 2, whereas linear ones afforded hexahy- dro-1,5-naphthyridines 3 . The former family of products presumably arises from a Michael addition-Mannich reaction sequence, while the latter one was formed via a Michael addition followed by double enamine formation by tautomerization. We assumed that these reactions are reversible and that the relative stability of the products depending on their substitution patterns could account for the observed selectivity.

When reinvestigating the scope of this reaction regarding carbocyclic $\beta$-keto esters and enals, it was realized that the structure of the latter substrate could also guide the chemoselectivity of the transformation. Herein, we wish to present our results on the substrate chemo-controlled Michael addition-initiated three-component synthesis of functionalized original aminobicyclo[3.2.1]octan-8-ones $\mathbf{4}^{5,6}$ and cycloalka[b]piperidines 5 (Scheme 1, new strategy), by studying the scope and limitations of both these threecomponent reactions.

Moreover, in line with our recent success in carrying out organocatalytic multicomponent syntheses of 2,6-diazabicyclo[2.2.2] octanones and pyrrolopiperazines, ${ }^{7-9}$ attempts to carry out the two new transformations in the presence of an organocatalyst will be discussed. Even though enantioselectivities remain low to moderate, our preliminary results highlight the intriguing possibility to obtain either one or the other family of products from the same starting materials depending on the selected organocatalyst. ${ }^{10}$

\section{Three-Component Synthesis of Aminobicyc-}

\section{lo[3.2.1]octan-8-ones}

The investigations were started by exposing ethyl 2oxocyclopentanecarboxylate $(\mathbf{6 a})$ to acrolein and primary amines under our standard conditions: $4 \AA$ MS in refluxing toluene (Scheme 2). As expected from the results of our seminal study, ${ }^{4} \alpha$-branched primary amines such as isopro- 


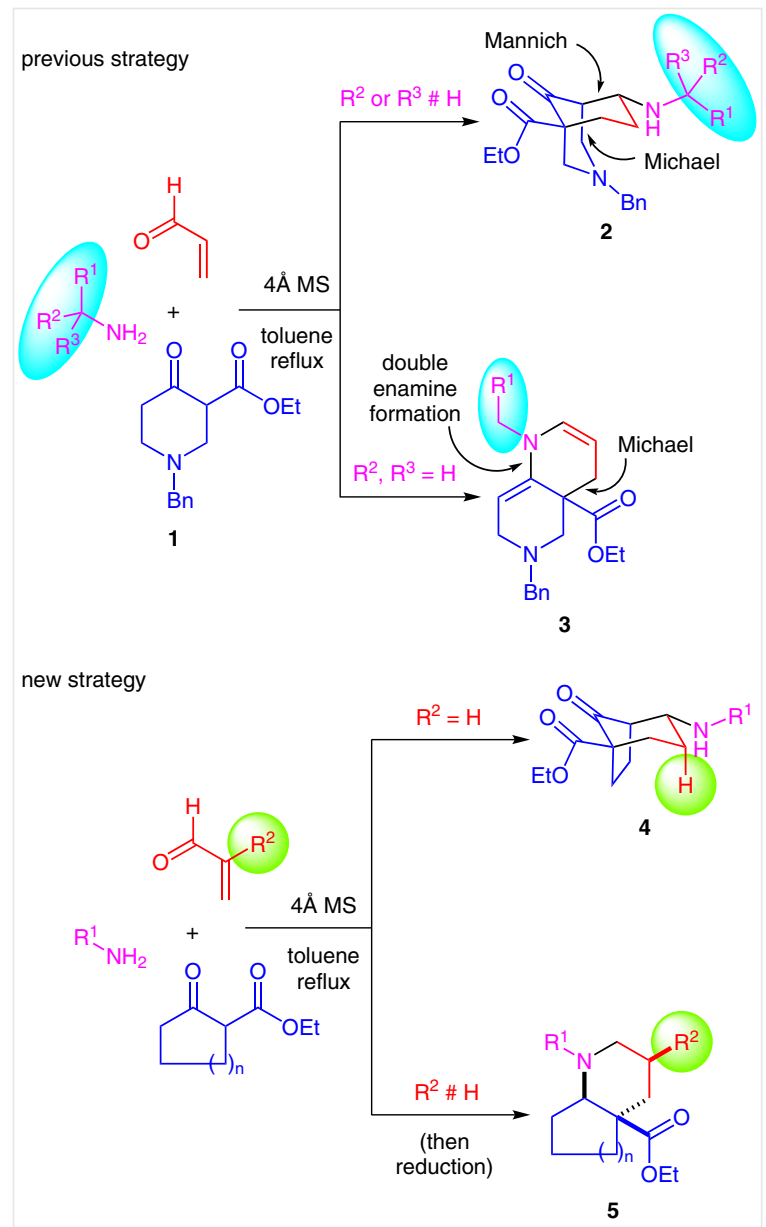

Scheme 1 Chemodivergent three-component reactions: previous and new strategies

pylamine (7a), cyclohexylamine (7b), or tert-butylamine (7c) exclusively furnished bicyclic products $\mathbf{4 a - c}$ with yields higher than $70 \%$ and modest diastereoselectivities. More surprisingly, the use of a variety of linear primary amines $\mathbf{7 d - h}$ also resulted in the formation of the bicyclic products $\mathbf{4 d - h}$ with the same efficiency. Different synthetically interesting motives such as allyl groups and (hetero)aromatics on the amine had no influence on the outcome of the reaction.

Among the three stereogenic centers of the bicyclic products $\mathbf{4 a}-\mathbf{h}$, the relative configuration of the two bridgehead ones being fixed, only the determination of the relative configuration of the stereogenic center that bears the amino group was required. After complete assignment of the signals on the ${ }^{1} \mathrm{H}$ and ${ }^{13} \mathrm{C}$ NMR spectra of product $\mathbf{4 d}$, thanks to 2D NMR studies, we could notice that the hydrogen atom located on this position appears at $3.03 \mathrm{ppm}$ and possesses one ${ }^{3} J_{a x-a x}$ coupling constant of $11.4 \mathrm{~Hz}$, indicating that the amino group is located on equatorial position (Figure 1). This observation is in accordance with our work on the corresponding bicyclic structures bearing an alcohol instead of the amine function ${ }^{11}$

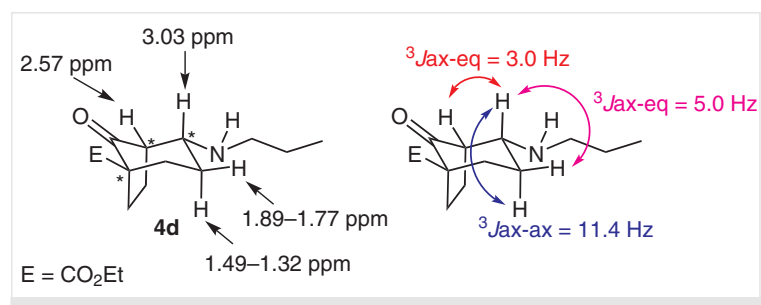

Figure 1 Assignment of the relative configurations in the aminobicyclo[3.2.1] octan-8-one 4d

\section{dines}

Three-Component Synthesis of Cycloalka[b]piperi-

When replacing acrolein by an $\alpha$-substituted enal, such as methacrolein $(\mathbf{8 a})$, a change in the chemoselectivity of the reaction was observed (Scheme 3).

After the initial Michael addition, presumably because of the additional steric hindrance brought by the $\alpha$-substituent, the reaction did not deliver the bridged bicyclic products, but rather proceeded towards the formation of dieneamines by addition of the nitrogen atom to the two carbon$\mathrm{yl}$ groups of the aldehyde and the ketone, respectively, followed by dehydration. Because of the low stability of these dieneamines, they were directly reduced by treatment with $\mathrm{NaBH}_{4}$, in a THF-AcOH-MeOH mixture to furnish the perhydrocycloalka[b]pyridines $\mathbf{5 a} \mathbf{a}-\mathbf{i}$, with moderate to high diastereoselectivities. Pleasingly, a diversity of linear aliphatic primary amines participated in this reaction with high efficiency to deliver the product $\mathbf{5 a}-\mathbf{e}$ in high yields. On the contrary, $\alpha$-branched primary amines were not suitable substrates in this reaction. Moreover, in addition to methacrolein, enals bearing ethyl or benzyl groups in the $\alpha$-position were also suitable substrates, even though products $\mathbf{5 f}$ and $\mathbf{5 g}$ were isolated in slightly reduced yields. To finish with the scope of the study, we could also show that a 6 -membered cyclic $\beta$-keto ester was also able to efficiently deliver the perhydroquinolines $\mathbf{5 h}$ and $\mathbf{5 i}$.

Once again, the products obtained in this second multicomponent reaction possess three stereogenic centers, the relative configuration of which was determined by analyzing the coupling constants in ${ }^{1} \mathrm{H}$ NMR spectra as shown for compounds $\mathbf{5 b}$ and $\mathbf{5 h}$, respectively (Figure 2). 


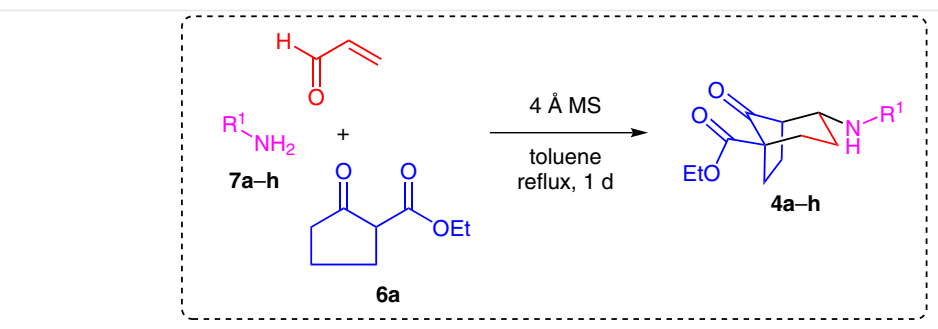

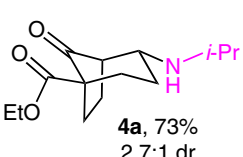

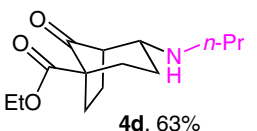

$2.3 .1 \mathrm{dr}$

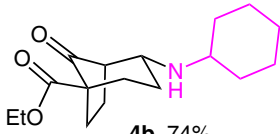
$2.6: 1 \mathrm{dr}$

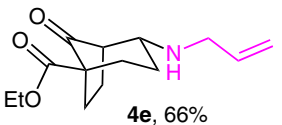

$2.4: 1 \mathrm{dr}$
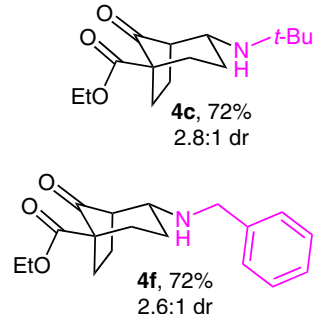

2.6:1 dr
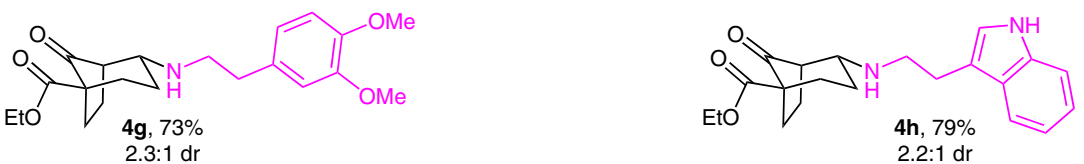

Scheme 2 Three-component synthesis of oxobicyclo[3.2.1]octanes

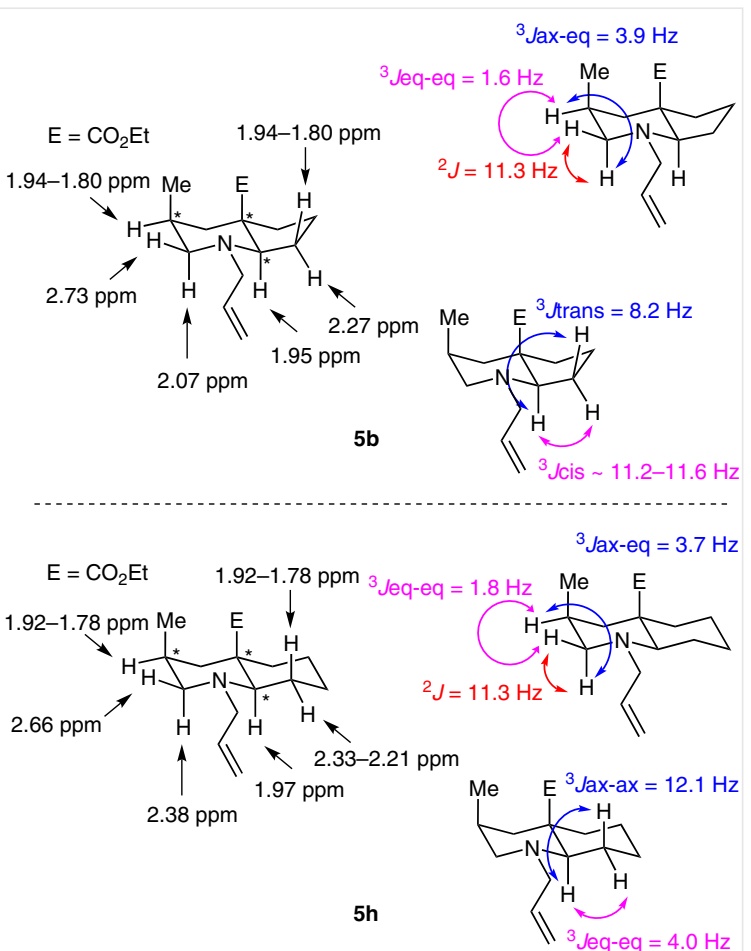

Figure 2 Assignment of the relative configurations of cycloalka[b]piperidines $\mathbf{5 b}, \mathbf{h}$
Both for the 5,6- and 6,6-bicyclic systems, the proton on the carbon atom bearing the methyl group exhibits no characteristic ${ }^{3} J_{a x-a x}$ coupling constant, indicating that it is located in equatorial position. As a consequence, the alkyl group on the heterocycle is set in axial position. On the opposite, the proton at the ring junction is placed in axial position and so is the ester group, indicating the rings are fused with a trans junction.

Proposed Mechanism: Origin of the Chemoselectivity

At first, it should be noted that, even though many of the steps involved in these two reaction pathways are likely to be reversible, the two products do not interconvert as their reexposure to the reaction conditions did not lead to any evolution. Based on the previous work in this field, ${ }^{4}$ we propose that the reaction is initiated by the Michael addition of the 1,3-keto ester to the enal, followed by imine formation on the resulting aliphatic aldehyde, to deliver the key intermediate A (Scheme 4). When $\mathrm{R}^{2}=\mathrm{H}$, after ketoenol tautomerism to form $\mathbf{B}$, the imine can undergo an intramolecular Mannich reaction to deliver the bridged bicyclic product 4 . On the contrary, we can surmise that the added steric hindrance provided by the substituent $R^{2}$ when it is different from an hydrogen atom, is responsible for the shift in chemoselectivity, by preventing the Mannich reaction or making it reversible under the reaction conditions. In a complementary manner, the $\mathrm{R}^{2}$ substituent will also favor the imine-enamine tautomerism towards intermediate $\mathbf{C}$, by increasing the stability of the more substitut- 
ed enamine. Consequently, the cyclization can occur this time by nucleophilic addition of the nitrogen atom to the ketone. Subsequent dehydration furnishes $\mathbf{D}$, which is reduced to allow isolating the final product $\mathbf{5}$. The higher stability of the more substituted dieneamine $\mathbf{D}$ when $\mathrm{R}^{2}$ is an alkyl chain can be another possible explanation for the chemoselectivity.

Organocatalyst-Controlled Chemodivergent ThreeComponent Reaction: Preliminary Results

In the recent years, organocatalysis has emerged as a very efficient tool to perform enantioselective multicomponent reactions. ${ }^{9}$ For this reason, our attention was turned towards the possibility to combine a cyclic $\beta$-keto ester, methacrolein, and allylamine in the presence of an organocatalyst to try to form enantioenriched polyheterocyclic products (Table 1 ).

As soon as the screening was started, it became clear that either family of product could be exclusively obtained in a highly chemoselective manner from the same starting materials depending on whether covalent ${ }^{12}$ or noncovalent ${ }^{13}$ organocatalysts were used. Proline-derived secondary amine $\mathbf{I}^{14}$ did not allow the formation of any of the two products (Table 1, entry 1). Assuming that a reason for this lack of reactivity was the high steric hindrance of methacrolein (8a), we turned our attention towards primary amines. Even though cinchona-alkaloid derived catalyst $\mathbf{I I}^{15}$ was inefficient (entry 2), bifunctional thiourea-primary amine III $^{16}$ enabled the chemoselective formation of the fused bicyclic product (entry 3 ). After reduction, perhydrocyclopenta $[b]$ pyridine $\mathbf{5 b}$ was isolated in $65 \%$ yield with an encouraging $45 \%$ enantiomeric excess. Unfortunately, reducing the reaction temperature to $0{ }^{\circ} \mathrm{C}$ both decreased the yield and the enantiomeric excess (entry 4). Although addition of $4 \AA$ MS to the reaction mixture had a positive effect on the yield, it resulted in an absolutely non-enantioselective transformation (entry 5 ). The use of the corresponding squaramide $\mathbf{I V}^{17}$ could not improve the results (entry 6 ).

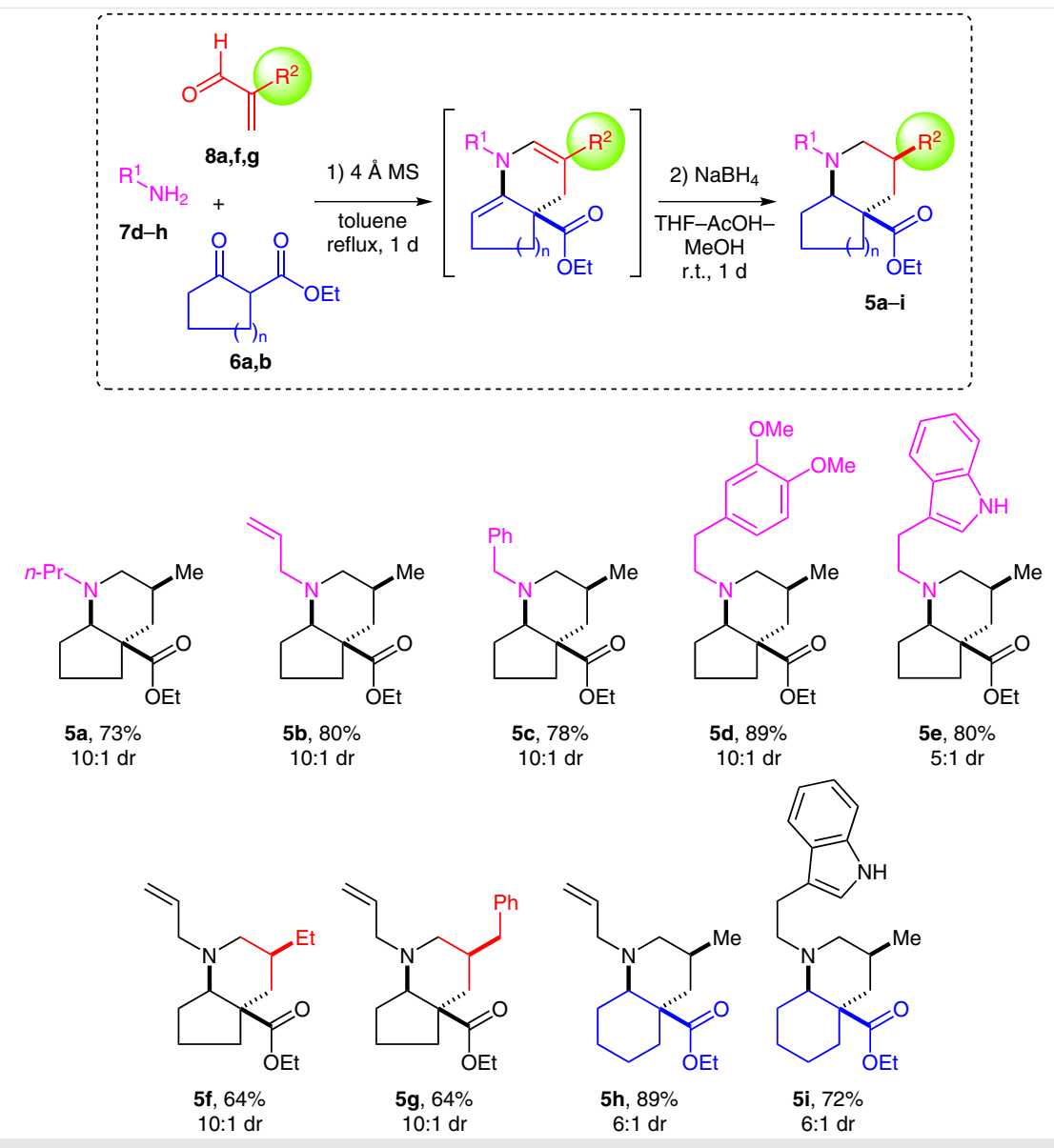

Scheme 3 Three-component synthesis of cycloalka[b]piperidines 
Table 1 Screening of organocatalysts in the three-component reaction ${ }^{\mathrm{a}}$
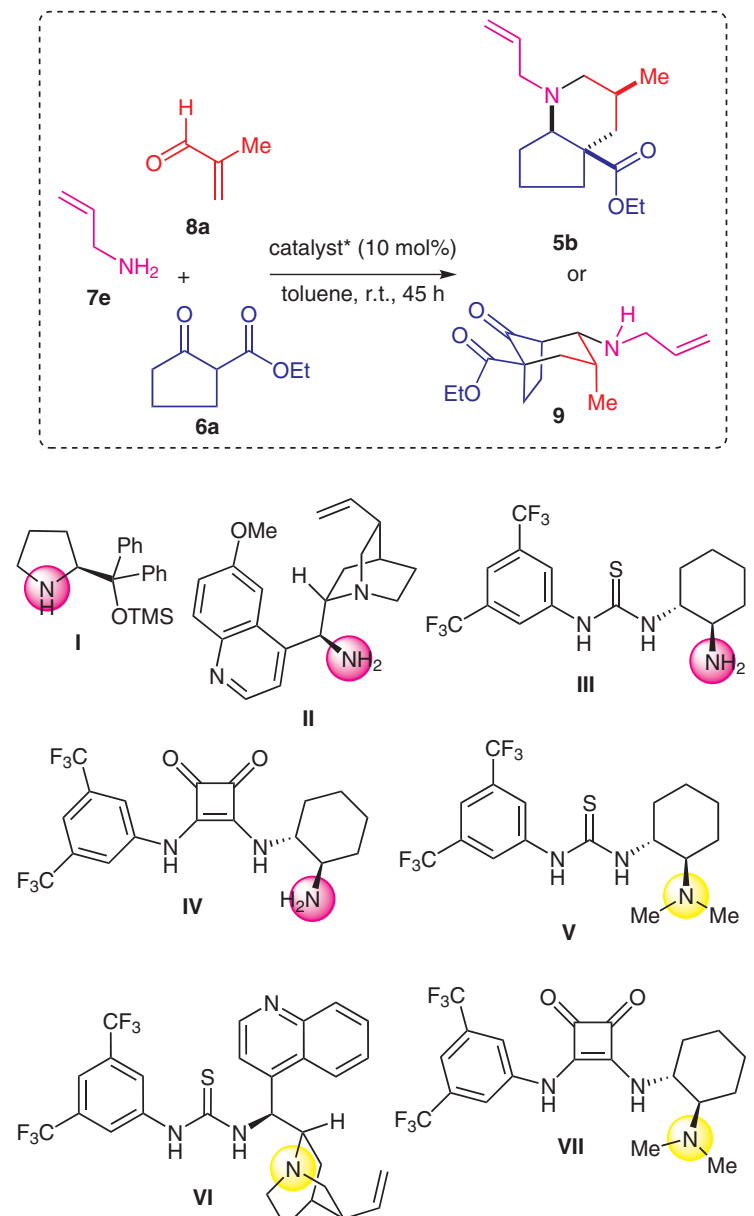

\begin{tabular}{llll}
\hline Entry & Catalyst & Product (yield) & ee (\%) \\
\hline $1^{\text {d }}$ & I & $\mathbf{5 b}$ (trace) & n.d. \\
$2^{\text {d }}$ & II & no product & n.d. \\
$3^{\text {d }}$ & III & $\mathbf{5 b}(65 \%)$ & 45 \\
$4^{\text {d,e }}$ & III & $\mathbf{5 b}(52 \%)$ & 37 \\
$5^{\text {d,f }}$ & III & $\mathbf{5 b}(77 \%)$ & 0 \\
$6^{\text {d }}$ & IV & $\mathbf{5 b}(18 \%)$ & 12 \\
$7^{f}$ & V & $\mathbf{9}(60 \%)$ & 6 \\
8 & VI & $\mathbf{9}(42 \%)$ & n.d. \\
9 & VII & $\mathbf{9}(34 \%)$ & n.d. \\
\hline
\end{tabular}

${ }^{a}$ General reaction conditions: ethyl 2-oxocyclopentanecarboxylate (6a; 50 mg, 1 equiv), methacrolein (8a; 1.2 equiv), allylamine (7e; 1 equiv) and organocatalyst I-VII (10 mol\%) were stirred at r.t. in toluene $(5 \mathrm{~mL})$ for $45 \mathrm{~h}$.

${ }^{b}$ Products were identified in the crude reaction mixture by ${ }^{1} \mathrm{H}$ NMR spectroscopy. Yields are given for pure product isolated by silica gel chromatography.

CEnantiomeric excesses were determined by HPLC on chiral stationary phase.

${ }^{d}$ After analysis of the crude reaction mixture, reduction $\left[\mathrm{NaBH}_{4}\right.$ in THF-AcOH-MeOH (4:2:1) at r.t. for $\left.24 \mathrm{~h}\right]$ was performed to allow the isolation of the pure

product 9.

Reaction performed at $0{ }^{\circ} \mathrm{C}$.

Powdered 4 A molecular sieves $(200 \mathrm{mg}$ ) were added to the organocatalytic reaction. 


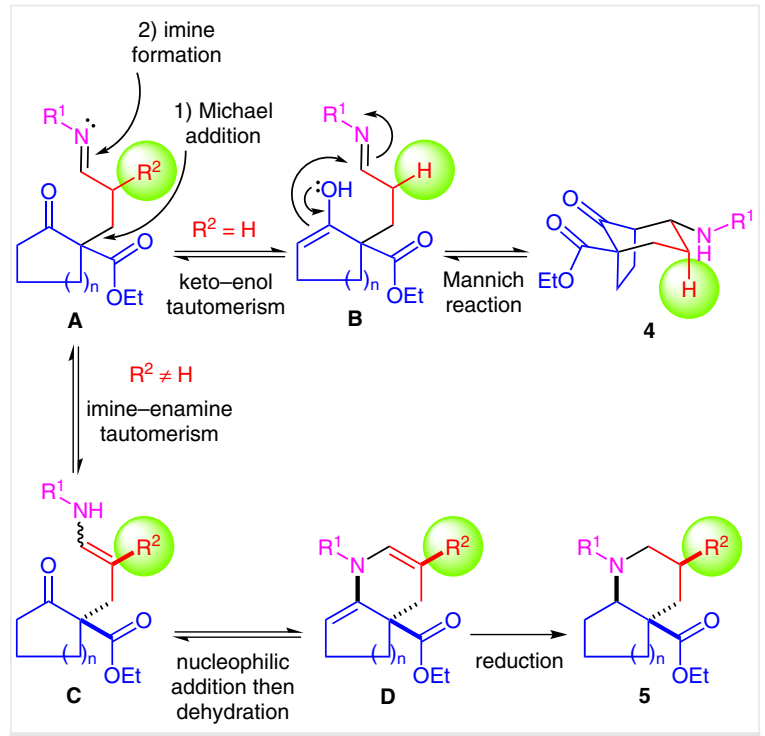

Scheme 4 Proposed mechanisms for the two multicomponent reactions

A series of tertiary amine-based noncovalent bifunctional catalysts were then evaluated: both thioureas $\mathbf{V}^{18}$ and VI, ${ }^{19}$ along with squaramide VII, ${ }^{20}$ did not deliver the expected compound $\mathbf{5 b}$, but rather the bridged bicycle $\mathbf{9}$ as the only product in $34-60 \%$ yields (entries 7-9). This new bicyclic product was formed as only one diastereomer. The relative configuration of the additional stereogenic center could be assigned by noticing the disappearance of ${ }^{3} J_{a x-a x}$ coupling for the proton on the carbon atom $\alpha$ to the nitrogen atom (Figure 3 ). Unfortunately, this product exhibited low stability in the HPLC conditions when attempting to evaluate its enantiomeric excess. Moreover, the only measure on product 9 formed in the conditions of entry 7 showed that it was virtually racemic. ${ }^{21}$

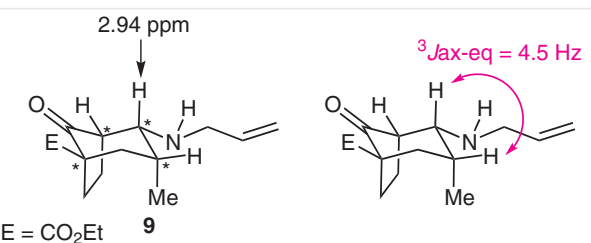

Figure 3 Assignment of the relative configurations of product 9

In conclusion, we have taken advantage of the reactivity of $\beta$-keto esters in Michael additions with enals to develop multicomponent synthetic routes towards two original bicyclic scaffolds: aminobicyclo[3.2.1]octan-8-ones 4 and cycloalka[b]piperidines $\mathbf{5}$. We could show that the presence of an $\alpha$-substituent on the enal was crucial for the chemoselectivity of the reaction, helping to direct it towards the formation of the latter heterocyclic systems. A series of organocatalysts were evaluated to perform enantioselective syntheses of these new scaffolds. Even though enantioselectivities remained low, we uncovered an intriguing possibility to obtain one or the other family of products from the same starting materials depending on the mode of action of the organocatalyst. Our next efforts will now focus on extending the possibility to carry out chemo- and enantioselective reactions using organocatalysis.

All commercially available reagents and solvents were used as received, except liquid enals that were distilled before use. 2-Benzylacrylaldehyde $(\mathbf{8 g})$ was prepared in one step from hydrocinnamaldehyde according to the literature. ${ }^{22}$ Toluene and THF were dried using a M-Braun SPS-800 system. Petroleum ether (PE) refers to a hydrocarbon mixture with a boiling range of $40-60{ }^{\circ} \mathrm{C}$.

Analytical TLC was performed on silica gel 60 F254 aluminum plates (Macherey-Nagel) containing a $254 \mathrm{~nm}$ fluorescent indicator. TLC plates were visualized by exposure to short wave ultraviolet light $(254 \mathrm{~nm})$ and to vanillin $\left(2 \mathrm{~g}\right.$ of vanillin and $4 \mathrm{~mL}$ of concd $\mathrm{H}_{2} \mathrm{SO}_{4}$ in $100 \mathrm{~mL}$ of EtOH) or $\mathrm{KMnO}_{4}\left(1.5 \mathrm{~g}\right.$ of $\mathrm{KMnO}_{4}, 10 \mathrm{~g}$ of $\mathrm{K}_{2} \mathrm{CO}_{3}$, and 1.25 $\mathrm{mL}$ of $10 \% \mathrm{NaOH}$ in $200 \mathrm{~mL}$ of $\mathrm{H}_{2} \mathrm{O}$ ) followed by heating. Flash column chromatography was performed using silica gel $(35-70 \mu \mathrm{m}, 60 \AA$ Acros).

${ }^{1} \mathrm{H}$ NMR spectra were recorded on a Bruker AV 400 or Bruker AV 300 spectrometer. Proton chemical shifts are reported in parts per million ( $\delta$ scale), and are referenced using residual protium in the NMR solvent $\left[\mathrm{CDCl}_{3}: \delta=7.26\left(\mathrm{CHCl}_{3}\right)\right] .{ }^{13} \mathrm{C}$ NMR spectra were recorded on a Bruker AV 400 or Bruker AV 300 spectrometer. Carbon chemical shifts are reported in parts per million ( $\delta$ scale), and are referenced using the carbon resonance of the solvent $\left[\delta=77.16\left(\mathrm{CHCl}_{3}\right)\right]$. Data are reported as follows: chemical shift $\left(\mathrm{CH}_{n}\right.$ where $\mathrm{n}$ is the number of hydrogen atoms linked to the carbon atom). HPLC analyses for the determination of enantiomeric excesses were performed on a MerckHitachi system equipped with the following chiral columns: Chiralpak AD-H, (S,S)-Whelk-O1. High-resolution mass spectra (HRMS) were recorded on a Waters Synapt G2 HDMS apparatus using a positive electrospray (ESI) ionization source.

\section{Aminobicyclo[3.2.1]octan-8-ones 4a-h; General Procedure}

A $100 \mathrm{~mL}$ two-necked flask filled with argon was equipped with a reflux condenser and a magnetic stirrer bar. Toluene $(25 \mathrm{~mL})$ was then added followed by commercially available activated $4 \AA$ MS (6 g). Then, ethyl 2-oxocyclopentanecarboxylate (6a; $200 \mathrm{mg}, 185 \mu \mathrm{L}, 1.28$ mmol, 1.0 equiv) was added followed by acrolein $(123 \mu \mathrm{L}, 1.53 \mathrm{mmol}$, 1.2 equiv), and the appropriate primary amine $\mathbf{7 a}-\mathbf{h}(1.28 \mathrm{mmol}, 1.0$ equiv). After complete conversion of $\mathbf{6 a}$, checked by TLC (eluent: see individual protocols below), the solution was filtered through a short pad of Celite, which was thoroughly washed with toluene. The solvent was evaporated under reduced pressure to obtain the crude compound, which was analyzed by ${ }^{13} \mathrm{C}$ NMR spectroscopy to measure the diastereomeric ratio. Purification by flash column chromatography on silica gel generally provided the pure product as a single diastereomer. 
Ethyl 4-(Isopropylamino)-8-oxobicyclo[3.2.1]octane-1-carboxylate (4a)

Prepared according to the general procedure using $i-\mathrm{PrNH}_{2}(\mathbf{7 a} ; 75.7$ $\mathrm{mg}, 110.0 \mu \mathrm{L}, 1.28 \mathrm{mmol}, 1.0$ equiv). The reaction was run for $24 \mathrm{~h}$ and the crude product was obtained with a dr of 2.7:1. Purification by flash chromatography (PE-EtOAc-Et $\left.{ }_{3} \mathrm{~N}, 3: 1: 0.025\right)$ afforded $4 a$ as a yellow oil; yield: $236 \mathrm{mg}(0.934 \mathrm{mmol}, 73 \%) ; R_{f}=0.15$ (PE-EtOAc$\mathrm{Et}_{3} \mathrm{~N}, 3: 1: 0.025$; detection: $\mathrm{KMnO}_{4}$ ).

${ }^{1} \mathrm{H}$ NMR (300 MHz, $\mathrm{CDCl}_{3}$ ): $\delta=4.16$ (q, $J=7.1 \mathrm{~Hz}, 2 \mathrm{H}$ ), 3.14 (ddd, $J=$ 11.3, 4.8, $2.6 \mathrm{~Hz}, 1 \mathrm{H}), 2.78(\mathrm{dt}, J=12.4,6.2 \mathrm{~Hz}, 1 \mathrm{H}), 2.61-2.43(\mathrm{~m}, 2$ $\mathrm{H}), 2.04(\mathrm{td}, J=13.6,5.3 \mathrm{~Hz}, 1 \mathrm{H}), 1.93-1.74(\mathrm{~m}, 5 \mathrm{H}), 1.39$ (tdd, $J=$ 13.8, 11.6, $6.2 \mathrm{~Hz}, 1 \mathrm{H}), 1.22$ (t, $J=7.1 \mathrm{~Hz}, 3 \mathrm{H}$ ), 0.97 (dd, $J=6.1,4.0 \mathrm{~Hz}$, $6 \mathrm{H})$; $\mathrm{NH}$ could not be assigned unambiguously.

${ }^{13} \mathrm{C}$ NMR (75 MHz, $\left.\mathrm{CDCl}_{3}\right): \delta=213.0(\mathrm{C}), 171.4(\mathrm{C}), 61.2\left(\mathrm{CH}_{2}\right), 60.0$ $(\mathrm{CH}), 57.2(\mathrm{C}), 50.3(\mathrm{CH}), 44.9(\mathrm{CH}), 33.3\left(\mathrm{CH}_{2}\right), 27.4\left(\mathrm{CH}_{2}\right), 26.3\left(\mathrm{CH}_{2}\right)$, $24.1\left(\mathrm{CH}_{3}\right), 22.6\left(\mathrm{CH}_{3}\right), 16.7\left(\mathrm{CH}_{2}\right), 14.2\left(\mathrm{CH}_{3}\right)$.

HRMS (ESI): $m / z$ calcd for $\left[\mathrm{C}_{14} \mathrm{H}_{23} \mathrm{NO}_{3}+\mathrm{H}\right]^{+}$: 254.1751; found: 254.1754 .

Ethyl 4-(Cyclohexylamino)-8-oxobicyclo[3.2.1 ]octane-1-carboxylate (4b)

Prepared according to the general procedure using cyclohexylamine (7b; $127 \mathrm{mg}, 146 \mu \mathrm{L}, 1.28 \mathrm{mmol}, 1.0$ equiv). The reaction was run for $24 \mathrm{~h}$ and the crude product was obtained with a dr of 2.6:1. Purification by flash chromatography (PE-EtOAc-Et $\left.{ }_{3} \mathrm{~N}, 2: 1: 0.025\right)$ afforded 4b as a yellow oil; yield: $278 \mathrm{mg}(0.947 \mathrm{mmol}, 74 \%) ; R_{f}=0.14(\mathrm{PE}-$ EtOAc-Et ${ }_{3} \mathrm{~N}, 2: 1: 0.025$; detection: $\left.\mathrm{KMnO}_{4}\right)$.

${ }^{1} \mathrm{H}$ NMR $\left(300 \mathrm{MHz}, \mathrm{CDCl}_{3}\right): \delta=4.12(\mathrm{q}, J=7.1 \mathrm{~Hz}, 2 \mathrm{H}), 3.16$ (ddd, $J=$ 11.2, 4.7, $2.5 \mathrm{~Hz}, 1 \mathrm{H}$ ), 2.58-2.41 (m, $2 \mathrm{H}$ ), 2.35 (ddd, $J=10.3,7.1$, 3.6 $\mathrm{Hz}, 1 \mathrm{H}), 1.99(\mathrm{td}, J=13.6,5.7 \mathrm{~Hz}, 1 \mathrm{H}), 1.90-1.70(\mathrm{~m}, 6 \mathrm{H}), 1.62(\mathrm{~d}, J=$ $11.2 \mathrm{~Hz}, 3 \mathrm{H}$ ), 1.52 (d, $J=11.4 \mathrm{~Hz}, 1 \mathrm{H}$ ), 1.36 (ddd, $J=25.3,13.5,6.3 \mathrm{~Hz}$ $1 \mathrm{H}), 1.20(\mathrm{t}, J=7.1 \mathrm{~Hz}, 3 \mathrm{H}), 1.15-0.81(\mathrm{~m}, 5 \mathrm{H})$; NH could not be assigned unambiguously.

${ }^{13} \mathrm{C}$ NMR (75 MHz, $\left.\mathrm{CDCl}_{3}\right): \delta=213.0(\mathrm{C}), 171.3(\mathrm{C}), 61.0\left(\mathrm{CH}_{2}\right), 59.5$ $(\mathrm{CH}), 57.1(\mathrm{C}), 53.0(\mathrm{CH}), 50.4(\mathrm{CH}), 34.6\left(\mathrm{CH}_{2}\right), 33.3\left(\mathrm{CH}_{2}\right), 33.2\left(\mathrm{CH}_{2}\right)$, $27.3\left(\mathrm{CH}_{2}\right), 26.3\left(\mathrm{CH}_{2}\right), 26.0\left(\mathrm{CH}_{2}\right), 25.1\left(\mathrm{CH}_{2}\right), 24.8\left(\mathrm{CH}_{2}\right), 16.6\left(\mathrm{CH}_{2}\right)$, $14.1\left(\mathrm{CH}_{3}\right)$.

HRMS (ESI): $m / z$ calcd for $\left[\mathrm{C}_{17} \mathrm{H}_{27} \mathrm{NO}_{3}+\mathrm{H}\right]^{+}$: 294.2064; found: 294.2066 .

\section{Ethyl 4-(tert-Butylamino)-8-oxobicyclo[3.2.1]octane-1-carboxyl- ate (4c)}

Prepared according to the general procedure using $t-\mathrm{BuNH}_{2}$ (7c; 93.6 $\mathrm{mg}, 135 \mu \mathrm{L}, 1.28 \mathrm{mmol}, 1.0$ equiv). The reaction was run for $23 \mathrm{~h}$ and the crude product was obtained with a dr of 2.8:1. Purification by flash chromatography (PE-EtOAc-Et $\left.{ }_{3} \mathrm{~N}, 2: 1: 0.025\right)$ afforded $4 \mathbf{c}$ as a yellow oil; yield: $246 \mathrm{mg}(0.922 \mathrm{mmol}, 72 \%) ; R_{f}=0.53$ (PE-EtOAc$\mathrm{Et}_{3} \mathrm{~N}, 2: 1: 0.025$; detection: $\left.\mathrm{KMnO}_{4}\right)$.

${ }^{1} \mathrm{H}$ NMR (300 MHz, $\left.\mathrm{CDCl}_{3}\right): \delta=4.17(\mathrm{q}, J=7.1 \mathrm{~Hz}, 2 \mathrm{H}), 3.12(\mathrm{ddd}, J=$ $11.2,5.0,2.6 \mathrm{~Hz}, 1 \mathrm{H}), 2.59-2.43(\mathrm{~m}, 1 \mathrm{H}), 2.43-2.35$ (m, $1 \mathrm{H}), 2.07-$ $1.74(\mathrm{~m}, 6 \mathrm{H}), 1.46$ (ddd, $J=25.3,13.5,6.2 \mathrm{~Hz}, 1 \mathrm{H}), 1.24(\mathrm{t}, J=7.1 \mathrm{~Hz}, 3$ $\mathrm{H}), 1.03(\mathrm{~d}, J=5.1 \mathrm{~Hz}, 9 \mathrm{H})$; NH could not be assigned unambiguously. ${ }^{13} \mathrm{C} \mathrm{NMR}\left(75 \mathrm{MHz}, \mathrm{CDCl}_{3}\right): \delta=212.7(\mathrm{C}), 171.6(\mathrm{C}), 61.1\left(\mathrm{CH}_{2}\right), 57.5(\mathrm{C})$, 57.0 (CH), $54.8(\mathrm{CH}), 51.2(\mathrm{C}), 33.2\left(\mathrm{CH}_{2}\right), 30.0\left(3 \mathrm{CH}_{3}\right), 28.4\left(\mathrm{CH}_{2}\right), 27.3$ $\left(\mathrm{CH}_{2}\right), 17.2\left(\mathrm{CH}_{2}\right), 14.3\left(\mathrm{CH}_{3}\right)$.

HRMS (ESI): $m / z$ calcd for $\left[\mathrm{C}_{15} \mathrm{H}_{25} \mathrm{NO}_{3}+\mathrm{H}\right]^{+}$: 268.1907; found: 268.1912 .
Ethyl 8-0xo-4-(propylamino)bicyclo[3.2.1]octane-1-carboxylate (4d)

Prepared according to the general procedure using $n-\mathrm{PrNH}_{2}$ (7d; 75.7 $\mathrm{mg}, 105 \mu \mathrm{L}, 1.28 \mathrm{mmol}, 1.0$ equiv). The reaction was run for $24 \mathrm{~h}$ and the crude product was obtained with a $\mathrm{dr}$ of $2.3: 1$. Purification by flash chromatography (PE-EtOAc-Et $\left.{ }_{3} \mathrm{~N}, 3: 1: 0.025\right)$ afforded $4 d$ as a yellow oil; yield: $204 \mathrm{mg}(0.806 \mathrm{mmol}, 63 \%) ; R_{f}=0.42$ (PE-EtOAc$\mathrm{Et}_{3} \mathrm{~N}, 3: 1: 0.025$; detection: UV, vanillin).

${ }^{1} \mathrm{H}$ NMR (400 MHz, $\mathrm{CDCl}_{3}$ ): $\delta=4.15$ (q, $J=7.1 \mathrm{~Hz}, 2 \mathrm{H}$ ), 3.03 (ddd, $J=$ 11.4, 5.0, $2.7 \mathrm{~Hz}, 1 \mathrm{H}), 2.57(\mathrm{dd}, J=6.1,3.0 \mathrm{~Hz}, 1 \mathrm{H}), 2.55-2.37(\mathrm{~m}, 3 \mathrm{H})$, 2.04 (tdd, $J=13.7,5.4,1.5 \mathrm{~Hz}, 1 \mathrm{H}), 1.91$ (dd, $J=6.2,1.3 \mathrm{~Hz}, 1 \mathrm{H}), 1.89$ $1.77(\mathrm{~m}, 4 \mathrm{H}), 1.49-1.32(\mathrm{~m}, 3 \mathrm{H}), 1.22(\mathrm{t}, J=7.1 \mathrm{~Hz}, 3 \mathrm{H}), 0.85(\mathrm{t}, J=7.4$ $\mathrm{Hz}, 3 \mathrm{H})$; NH could not be assigned unambiguously.

${ }^{13} \mathrm{C}$ NMR (101 MHz, $\left.\mathrm{CDCl}_{3}\right): \delta=213.1(\mathrm{C}), 171.4(\mathrm{C}), 63.0(\mathrm{CH}), 61.1$ $\left(\mathrm{CH}_{2}\right), 57.1(\mathrm{C}), 50.1(\mathrm{CH}), 48.7\left(\mathrm{CH}_{2}\right), 33.3\left(\mathrm{CH}_{2}\right), 27.4\left(\mathrm{CH}_{2}\right), 25.8$ $\left(\mathrm{CH}_{2}\right), 23.4\left(\mathrm{CH}_{2}\right), 16.6\left(\mathrm{CH}_{2}\right), 14.2\left(\mathrm{CH}_{3}\right), 11.7\left(\mathrm{CH}_{3}\right)$.

HRMS (ESI): $m / z$ calcd for $\left[\mathrm{C}_{14} \mathrm{H}_{23} \mathrm{NO}_{3}+\mathrm{H}\right]^{+}$: 254.1750; found: 254.1750 .

Ethyl 4-(Allylamino)-8-oxobicyclo[3.2.1]octane-1-carboxylate (4e) Prepared according to the general procedure using allylamine (7e; $73.1 \mathrm{mg}, 95.8 \mu \mathrm{L}, 1.28 \mathrm{mmol}, 1.0$ equiv). The reaction was run for $22 \mathrm{~h}$ and the crude product was obtained with a $\mathrm{dr}$ of $2.4: 1$. Purification by flash chromatography (PE-EtOAc-Et $\left.{ }_{3} \mathrm{~N}, 3: 1: 0.025\right)$ afforded $4 \mathbf{e}$ as a yellow oil; yield: $212 \mathrm{mg}(0.845 \mathrm{mmol}, 66 \%) ; R_{f}=0.24$ (PE-EtOAc$\mathrm{Et}_{3} \mathrm{~N}, 3: 1: 0.025$; detection: UV, vanillin).

${ }^{1} \mathrm{H}$ NMR (400 MHz, $\mathrm{CDCl}_{3}$ ): $\delta=5.83$ (ddt, $J=16.2,10.2,5.9 \mathrm{~Hz}, 1 \mathrm{H}$ ), 5.16 (ddd, $J=17.2,3.1,1.5 \mathrm{~Hz}, 1 \mathrm{H}$ ), 5.08 (ddd, $J=10.2,2.8,1.4 \mathrm{~Hz}, 1$ $\mathrm{H}), 4.19(\mathrm{q}, J=7.1 \mathrm{~Hz}, 2 \mathrm{H}), 3.33-3.04(\mathrm{~m}, 3 \mathrm{H}), 2.63-2.49(\mathrm{~m}, 2 \mathrm{H})$, 2.07 (tdd, $J=13.7,5.3,1.4 \mathrm{~Hz}, 1 \mathrm{H}), 1.97-1.83(\mathrm{~m}, 5 \mathrm{H}), 1.48$ (tdd, $J=$ $13.8,11.6,6.2 \mathrm{~Hz}, 1 \mathrm{H}), 1.26(\mathrm{t}, J=7.1 \mathrm{~Hz}, 3 \mathrm{H})$; $\mathrm{NH}$ could not be assigned unambiguously.

${ }^{13} \mathrm{C} \mathrm{NMR}\left(101 \mathrm{MHz}, \mathrm{CDCl}_{3}\right): \delta=212.9(\mathrm{C}), 171.5(\mathrm{C}), 136.5(\mathrm{CH}), 116.3$ $\left(\mathrm{CH}_{2}\right), 62.4(\mathrm{CH}), 61.3\left(\mathrm{CH}_{2}\right), 57.2(\mathrm{C}), 50.2(\mathrm{CH}), 49.5\left(\mathrm{CH}_{2}\right), 33.4\left(\mathrm{CH}_{2}\right)$, $27.4\left(\mathrm{CH}_{2}\right), 25.8\left(\mathrm{CH}_{2}\right), 16.8\left(\mathrm{CH}_{2}\right), 14.3\left(\mathrm{CH}_{3}\right)$.

HRMS (ESI): $m / z$ calcd for $\left[\mathrm{C}_{14} \mathrm{H}_{21} \mathrm{NO}_{3}+\mathrm{H}\right]^{+}:$252.1594; found: 252.1599 .

\section{Ethyl 4-(Benzylamino)-8-oxobicyclo[3.2.1]octane-1-carboxylate} (4f)

Prepared according to the general procedure using benzylamine (7f; $137 \mathrm{mg}, 140 \mu \mathrm{L}, 1.28 \mathrm{mmol}, 1.0$ equiv). The reaction was run for $24 \mathrm{~h}$ and the crude product was obtained with a dr of 2.6:1. Purification by flash chromatography (PE-EtOAc-Et $\left.{ }_{3} \mathrm{~N}, 2: 1: 0.025\right)$ afforded $\mathbf{4 f}$ as a yellow oil; yield: $278 \mathrm{mg}(0.922 \mathrm{mmol}, 72 \%) ; R_{f}=0.48$ (PE-EtOAc$\mathrm{Et}_{3} \mathrm{~N}, 2: 1: 0.025$; detection: UV, vanillin).

${ }^{1} \mathrm{H} \mathrm{NMR}\left(300 \mathrm{MHz}, \mathrm{CDCl}_{3}\right): \delta=7.33-7.25(\mathrm{~m}, 5 \mathrm{H}), 4.19(\mathrm{q}, J=7.1 \mathrm{~Hz}, 2$ $\mathrm{H}), 3.73(\mathrm{q}, J=13.2 \mathrm{~Hz}, 2 \mathrm{H}), 3.15$ (ddd, $J=11.3,5.0,2.7 \mathrm{~Hz}, 1 \mathrm{H}), 2.68-$ $2.61(\mathrm{~m}, 1 \mathrm{H}), 2.59-2.51(\mathrm{~m}, 1 \mathrm{H}), 2.06$ (ddd, $J=13.6,5.3,1.3 \mathrm{~Hz}, 1 \mathrm{H}$ ), 2.00-1.87 (m, $5 \mathrm{H}$ ), 1.50 (ddd, $J=25.3,13.5,6.3 \mathrm{~Hz}, 1 \mathrm{H}$ ), 1.25 (t, $J=7.1$ $\mathrm{Hz}, 3 \mathrm{H})$; NH could not be assigned unambiguously.

${ }^{13} \mathrm{C}$ NMR (75 MHz, $\mathrm{CDCl}_{3}$ ): $\delta=213.0$ (C), 171.5 (C), $140.1(\mathrm{C}), 128.6$ (2 $\mathrm{CH}), 128.1(2 \mathrm{CH}), 127.2(\mathrm{CH}), 62.5(\mathrm{CH}), 61.3\left(\mathrm{CH}_{2}\right), 57.2(\mathrm{C}), 51.0$ $\left(\mathrm{CH}_{2}\right), 50.1(\mathrm{CH}), 33.4\left(\mathrm{CH}_{2}\right), 27.4\left(\mathrm{CH}_{2}\right), 25.7\left(\mathrm{CH}_{2}\right), 16.8\left(\mathrm{CH}_{2}\right), 14.3$ $\left(\mathrm{CH}_{3}\right)$.

HRMS (ESI): $m / z$ calcd for $\left[\mathrm{C}_{18} \mathrm{H}_{23} \mathrm{NO}_{3}+\mathrm{H}\right]^{+}$: 302.1750; found: 302.1749 
Ethyl 4-[(3,4-Dimethoxyphenethyl)amino]-8-oxobicyclo[3.2.1]octane-1-carboxylate (4g)

Prepared according to the general procedure using 3,4-dimethoxyphenethylamine ( $\mathbf{7 g}$; $232 \mathrm{mg}, 217 \mu \mathrm{L}, 1.28 \mathrm{mmol}, 1.0$ equiv). The reaction was run for $24 \mathrm{~h}$ and the crude product was obtained with a dr of 2.2:1. Purification by flash chromatography (PE-EtOAc-Et $\mathrm{N}_{3}$ 2:1:0.025) afforded $\mathbf{4 g}$ as a yellow oil; yield: $379 \mathrm{mg}(1.010 \mathrm{mmol}$, $79 \%$ ); $R_{f}=0.06$ (PE-EtOAc-Et ${ }_{3} \mathrm{~N}, 2: 1: 0.025$; detection: UV, vanillin).

${ }^{1} \mathrm{H} \mathrm{NMR}\left(300 \mathrm{MHz}, \mathrm{CDCl}_{3}\right): \delta=6.75-6.68(\mathrm{~m}, 1 \mathrm{H}), 6.67-6.60(\mathrm{~m}, 2 \mathrm{H})$, $4.10(\mathrm{q}, J=7.1 \mathrm{~Hz}, 2 \mathrm{H}), 3.77$ (d, $J=3.8 \mathrm{~Hz}, 6 \mathrm{H}), 3.00$ (ddd, $J=11.3,4.8$, $2.5 \mathrm{~Hz}, 1 \mathrm{H}), 2.82-2.53(\mathrm{~m}, 4 \mathrm{H}), 2.53-2.35(\mathrm{~m}, 2 \mathrm{H}), 2.11-1.67(\mathrm{~m}, 6$ H), 1.35 (ddd, $J=18.9,13.6,6.8 \mathrm{~Hz}, 1 \mathrm{H}), 1.18(\mathrm{t}, J=7.1 \mathrm{~Hz}, 3 \mathrm{H}) ; \mathrm{NH}$ could not be assigned unambiguously.

${ }^{13} \mathrm{CNMR}\left(75 \mathrm{MHz}, \mathrm{CDCl}_{3}\right): \delta=212.8$ (C), $171.2(\mathrm{C}), 148.8(\mathrm{C}), 147.4(\mathrm{C})$, $132.1(\mathrm{C}), 120.4(\mathrm{CH}), 111.8(\mathrm{CH}), 111.3(\mathrm{CH}), 62.7(\mathrm{CH}), 61.0\left(\mathrm{CH}_{2}\right)$ $56.9(\mathrm{C}), 55.8\left(\mathrm{CH}_{3}\right), 55.7\left(\mathrm{CH}_{3}\right), 49.8(\mathrm{CH}), 47.8\left(\mathrm{CH}_{2}\right), 35.7\left(\mathrm{CH}_{2}\right), 33.1$ $\left(\mathrm{CH}_{2}\right), 27.1\left(\mathrm{CH}_{2}\right), 25.5\left(\mathrm{CH}_{2}\right), 16.4\left(\mathrm{CH}_{2}\right), 14.0\left(\mathrm{CH}_{3}\right)$.

HRMS (ESI): $\mathrm{m} / z$ calcd for $\left[\mathrm{C}_{21} \mathrm{H}_{29} \mathrm{~N}_{2} \mathrm{O}_{5}+\mathrm{H}\right]^{+}: 376.2018$; found: 376.2119.

\section{Ethyl 4-\{[2-(1H-Indol-3-yl)ethyl]amino\}-8-oxobicyclo[3.2.1]oc- tane-1-carboxylate (4h)}

Prepared according to the general procedure using tryptamine ( $\mathbf{7 h}$; $205 \mathrm{mg}, 1.28 \mathrm{mmol}, 1.0$ equiv). The reaction was run for $23 \mathrm{~h}$ and the crude product was obtained with a dr of 2.3:1. Purification by flash chromatography (PE-EtOAc, 2:1) afforded $\mathbf{4 h}$ as a yellow oil; yield: $331 \mathrm{mg}$ (0.934 mmol, 73\%); $R_{f}=0.05$ (PE-EtOAc, 2:1; detection: UV, vanillin).

${ }^{1} \mathrm{H} \mathrm{NMR}\left(300 \mathrm{MHz}, \mathrm{CDCl}_{3}\right): \delta$ (major diastereomer) $=8.36(\mathrm{~s}, 1 \mathrm{H})$ 7.64-7.54 (m, $1 \mathrm{H}), 7.39-7.30(\mathrm{~m}, 1 \mathrm{H}), 7.22-7.16(\mathrm{~m}, 2 \mathrm{H}), 7.03-6.96$ $(\mathrm{m}, 1 \mathrm{H}), 4.29-4.13(\mathrm{~m}, 2 \mathrm{H}), 3.10$ (ddd, $J=11.4,5.0,2.6 \mathrm{~Hz}, 1 \mathrm{H}), 3.03-$ $2.81(\mathrm{~m}, 4 \mathrm{H}), 2.62-2.41(\mathrm{~m}, 2 \mathrm{H}), 2.15-1.52(\mathrm{~m}, 7 \mathrm{H}), 1.49-1.35(\mathrm{~m}, 1$ $\mathrm{H}), 1.30-1.23(\mathrm{~m}, 3 \mathrm{H}) ; \delta($ minor diastereomer $)=8.27(\mathrm{~s}, 1 \mathrm{H}), 7.64$ $7.54(\mathrm{~m}, 1 \mathrm{H}), 7.39-7.30(\mathrm{~m}, 1 \mathrm{H}), 7.22-7.16(\mathrm{~m}, 2 \mathrm{H}), 7.03-6.96$ (m, 1 $\mathrm{H}), 4.29-4.13(\mathrm{~m}, 2 \mathrm{H}), 3.23-3.14(\mathrm{~m}, 1 \mathrm{H}), 3.03-2.81(\mathrm{~m}, 4 \mathrm{H}), 2.62-$ $2.41(\mathrm{~m}, 2 \mathrm{H}), 2.15-1.52(\mathrm{~m}, 7 \mathrm{H}), 1.49-1.35(\mathrm{~m}, 1 \mathrm{H}), 1.30-1.23(\mathrm{~m}, 3$ $\mathrm{H})$.

${ }^{13} \mathrm{C}$ NMR $\left(75 \mathrm{MHz}, \mathrm{CDCl}_{3}\right)$ : $\delta$ (major diastereomer) $=213.3(\mathrm{C}), 171.5$ (C), $136.5(\mathrm{C}), 127.4(\mathrm{C}), 122.3(\mathrm{CH}), 122.0(\mathrm{CH}), 119.4(\mathrm{CH}), 118.7$ (CH), $113.3(\mathrm{C}), 111.3(\mathrm{CH}), 62.9(\mathrm{CH}), 61.4\left(\mathrm{CH}_{2}\right), 57.2(\mathrm{C}), 50.0(\mathrm{CH})$, 46.7 $\left(\mathrm{CH}_{2}\right), 33.3\left(\mathrm{CH}_{2}\right), 27.3\left(\mathrm{CH}_{2}\right), 25.8\left(\mathrm{CH}_{2}\right), 25.6\left(\mathrm{CH}_{2}\right), 16.5\left(\mathrm{CH}_{2}\right)$, $14.2\left(\mathrm{CH}_{3}\right) ; \delta$ (minor diastereomer) $=212.5(\mathrm{C}), 171.5(\mathrm{C}), 136.4(\mathrm{C})$, 127.4 (C), $122.4(\mathrm{CH}), 121.8(\mathrm{CH}), 119.1(\mathrm{CH}), 118.7(\mathrm{CH}), 113.6(\mathrm{C})$, $111.3(\mathrm{CH}), 65.1(\mathrm{CH}), 61.3\left(\mathrm{CH}_{2}\right), 57.5(\mathrm{C}), 49.8(\mathrm{CH}), 47.3\left(\mathrm{CH}_{2}\right), 34.3$ $\left(\mathrm{CH}_{2}\right), 26.3\left(\mathrm{CH}_{2}\right), 25.7\left(\mathrm{CH}_{2}\right), 23.3\left(\mathrm{CH}_{2}\right), 20.4\left(\mathrm{CH}_{2}\right), 14.2\left(\mathrm{CH}_{3}\right)$.

HRMS (ESI): $m / z$ calcd for $\left[\mathrm{C}_{21} \mathrm{H}_{26} \mathrm{~N}_{2} \mathrm{O}_{3}+\mathrm{H}\right]^{+}: 355.2016$; found: 355.2021.

\section{Cycloalka[b]piperidines 5a-i; General Procedure}

A $100 \mathrm{~mL}$ two-necked flask filled with argon was equipped with a reflux condenser and a magnetic stirrer bar. Toluene $(25 \mathrm{~mL})$ was then added followed by commercially available activated 4 Å MS (6 g). Then, the $\beta$-keto ester $\mathbf{6 a}, \mathbf{h}$ ( 1.28 or $1.17 \mathrm{mmol}, 1.0$ equiv) was added followed by the $\alpha, \beta$-unsaturated aldehyde $8(1.54$ or $1.41 \mathrm{mmol}, 1.2$ equiv), and the appropriate primary amine $\mathbf{7 d}-\mathbf{h}(1.28$ or $1.17 \mathrm{mmol}$, 1.0 equiv). After complete conversion of the $\beta$-keto ester, checked by TLC (eluent: see individual protocols below), the solution was filtered through a short pad of Celite, which was thoroughly washed with toluene. The solvent was evaporated under reduced pressure to obtain the crude dieneamine. In a $50 \mathrm{~mL}$ two-necked flask equipped with a magnetic stirring bar were added the crude dieneamine dissolved in THF, and $\mathrm{NaBH}_{4}$ (6 equiv) under argon. Then, $\mathrm{AcOH}$ was slowly added (caution: gas evolution), followed by EtOH. The resultant suspension was stirred at r.t. for $24 \mathrm{~h}$. After completion of the reaction, most of the solvent was removed under reduced pressure. $\mathrm{H}_{2} \mathrm{O}(15 \mathrm{~mL}), 10 \%$ aq $\mathrm{NaOH}(15 \mathrm{~mL})$, and $\mathrm{Et}_{2} \mathrm{O}(15 \mathrm{~mL})$ were added. The organic layer was separated and the aqueous phase was further extracted with $\mathrm{Et}_{2} \mathrm{O}(2 \times$ $15 \mathrm{~mL})$. The combined organic layers were then washed with $\mathrm{H}_{2} \mathrm{O}(2 \times$ $10 \mathrm{~mL})$, brine $(15 \mathrm{~mL})$, and dried $\left(\mathrm{Na}_{2} \mathrm{SO}_{4}\right)$. The solvent was evaporated giving the crude product, which was analyzed by ${ }^{13} \mathrm{C}$ NMR spectroscopy to measure the diastereomeric ratio. Purification by flash column chromatography on silica gel provided the pure product.

Ethyl 3-Methyl-1-propyloctahydro-1H-cyclopenta[b]pyridine-4acarboxylate (5a)

Prepared according to the general procedure using ethyl 2-oxocyclopentanecarboxylate (6a; $200 \mathrm{mg}, 185 \mu \mathrm{L}, 1.28 \mathrm{mmol}, 1.0$ equiv), methacrolein (8a; $108 \mathrm{mg}, 127 \mu \mathrm{L}, 1.54 \mathrm{mmol}, 1.2$ equiv), and $n$ $\mathrm{PrNH}_{2}$ (7d; $76 \mathrm{mg}, 105 \mu \mathrm{L}, 1.28 \mathrm{mmol}, 1.0$ equiv). The reduction was carried out with $\mathrm{NaBH}_{4}(265 \mathrm{mg})$ in THF $(5 \mathrm{~mL}), \mathrm{AcOH}(2.8 \mathrm{~mL})$, and EtOH (1.4 mL). The crude product was obtained with a dr of 10:1. Purification by flash chromatography (PE-EtOAc, 1:1) afforded $\mathbf{5 a}$ as a yellow oil; yield: $237 \mathrm{mg}$ ( $0.934 \mathrm{mmol}, 73 \%) ; R_{f}=0.06$ (PE-EtOAc, 1:1; detection: $\mathrm{KMnO}_{4}$ ).

${ }^{1} \mathrm{H} \mathrm{NMR}\left(300 \mathrm{MHz}, \mathrm{CDCl}_{3}\right): \delta=4.07(\mathrm{qd}, J=7.1,1.8 \mathrm{~Hz}, 2 \mathrm{H}), 2.75(\mathrm{~d}, J=$ $11.1 \mathrm{~Hz}, 1 \mathrm{H}), 2.61(\mathrm{ddd}, J=12.3,8.9,7.3 \mathrm{~Hz}, 1 \mathrm{H}), 2.42(\mathrm{~d}, J=13.1 \mathrm{~Hz}$ $1 \mathrm{H}), 2.24(\mathrm{ddd}, J=11.8,10.9,6.0 \mathrm{~Hz}, 1 \mathrm{H}), 2.17-2.07(\mathrm{~m}, 1 \mathrm{H}), 2.01$ $(\mathrm{dd}, J=11.1,3.7 \mathrm{~Hz}, 1 \mathrm{H}), 1.97-1.79(\mathrm{~m}, 3 \mathrm{H}), 1.81-1.70(\mathrm{~m}, 1 \mathrm{H}), 1.66-$ $1.53(\mathrm{~m}, 2 \mathrm{H}), 1.55-1.33(\mathrm{~m}, 3 \mathrm{H}), 1.31(\mathrm{dd}, J=13.1,5.4 \mathrm{~Hz}, 1 \mathrm{H}), 1.23$ $(\mathrm{t}, J=7.1 \mathrm{~Hz}, 3 \mathrm{H}), 0.95(\mathrm{~d}, J=7.4 \mathrm{~Hz}, 3 \mathrm{H}), 0.86(\mathrm{t}, J=7.3 \mathrm{~Hz}, 3 \mathrm{H})$.

${ }^{13} \mathrm{C}$ NMR $\left(75 \mathrm{MHz}, \mathrm{CDCl}_{3}\right): \delta=175.9(\mathrm{C}), 73.3(\mathrm{CH}), 60.3\left(\mathrm{CH}_{2}\right), 59.6$ $\left(\mathrm{CH}_{2}\right), 57.3\left(\mathrm{CH}_{2}\right), 50.9(\mathrm{C}), 40.4\left(\mathrm{CH}_{2}\right), 37.2\left(\mathrm{CH}_{2}\right), 29.5(\mathrm{CH}), 26.1$ $\left(\mathrm{CH}_{2}\right), 19.6\left(\mathrm{CH}_{3}\right), 19.5\left(\mathrm{CH}_{2}\right), 19.1\left(\mathrm{CH}_{2}\right), 14.2\left(\mathrm{CH}_{3}\right), 12.1\left(\mathrm{CH}_{3}\right)$.

HRMS (ESI): $m / z$ calcd for $\left[\mathrm{C}_{15} \mathrm{H}_{27} \mathrm{NO}_{2}+\mathrm{H}\right]^{+}: 254.2114$; found: 254.2113 .

\section{Ethyl 1-Allyl-3-methyloctahydro-1H-cyclopenta[b]pyridine-4a-} carboxylate (5b)

Prepared according to the general procedure using $\mathbf{6 a}(200 \mathrm{mg}, 185$ $\mu \mathrm{L}, 1.28 \mathrm{mmol}, 1.0$ equiv), methacrolein $(\mathbf{8 a} ; 108 \mathrm{mg}, 127 \mu \mathrm{L}, 1.54$ mmol, 1.2 equiv), and allylamine (7e; $73.1 \mathrm{mg}$, $95.8 \mu \mathrm{L}, 1.28 \mathrm{mmol}$, 1.0 equiv). The reduction was carried out with $\mathrm{NaBH}_{4}(318 \mathrm{mg})$ in THF $(6 \mathrm{~mL}), \mathrm{AcOH}(4.8 \mathrm{~mL})$, and EtOH $(2.4 \mathrm{~mL})$. The crude product was obtained with a dr of 10:1. Purification by flash chromatography (PEEtOAc, 1:3) afforded $\mathbf{5 b}$ as a yellow oil; yield: $257 \mathrm{mg}(1.02 \mathrm{mmol}$, $80 \%$ ); $R_{f}=0.05$ (PE-EtOAc, 1:3; detection: $\mathrm{KMnO}_{4}$ ).

${ }^{1} \mathrm{H} \mathrm{NMR}\left(400 \mathrm{MHz}, \mathrm{CDCl}_{3}\right): \delta=5.94(\mathrm{dddd}, J=17.4,10.2,7.7,5.3 \mathrm{~Hz}, 1$ H), 5.16 (ddd, $J=17.2,3.1,1.9 \mathrm{~Hz}, 1 \mathrm{H}$ ), 5.09 (ddd, $J=10.2,2.8,1.4 \mathrm{~Hz}$ $1 \mathrm{H}), 4.10(\mathrm{q}, J=7.1 \mathrm{~Hz}, 2 \mathrm{H}$ ), 3.40 (ddt, $J=13.9,5.3,1.6 \mathrm{~Hz}, 1 \mathrm{H}$ ), 2.87 $(\mathrm{dd}, J=13.9,7.7 \mathrm{~Hz}, 1 \mathrm{H}), 2.73(\mathrm{dt}, J=11.3,1.6 \mathrm{~Hz}, 1 \mathrm{H}), 2.44(\mathrm{dt}, J=$ $13.1,1.6 \mathrm{~Hz}, 1 \mathrm{H}$ ), 2.27 (tdd, $J=11.2,9.1,7.9 \mathrm{~Hz}, 1 \mathrm{H}$ ), 2.07 (dd, $J=11.3$, $3.9 \mathrm{~Hz}, 1 \mathrm{H}), 1.95(\mathrm{dd}, J=12.0,8.1 \mathrm{~Hz}, 1 \mathrm{H}), 1.94-1.80(\mathrm{~m}, 3 \mathrm{H}), 1.68-$ $1.59(\mathrm{~m}, 2 \mathrm{H}), 1.49-1.39(\mathrm{~m}, 1 \mathrm{H}), 1.32(\mathrm{dd}, J=13.2,5.5 \mathrm{~Hz}, 1 \mathrm{H}), 1.23$ $(\mathrm{t}, J=7.1 \mathrm{~Hz}, 3 \mathrm{H}), 0.96(\mathrm{~d}, J=7.4 \mathrm{~Hz}, 3 \mathrm{H})$.

$\left.{ }^{13} \mathrm{C} \mathrm{NMR} \mathrm{(101} \mathrm{MHz,} \mathrm{CDCl} 3\right): \delta=175.8(\mathrm{C}), 135.7(\mathrm{CH}), 117.1\left(\mathrm{CH}_{2}\right), 72.9$ (CH), $60.6\left(\mathrm{CH}_{2}\right), 59.6\left(\mathrm{CH}_{2}\right), 59.0\left(\mathrm{CH}_{2}\right), 50.8(\mathrm{C}), 40.2\left(\mathrm{CH}_{2}\right), 37.1$ $\left(\mathrm{CH}_{2}\right), 29.5(\mathrm{CH}), 26.0\left(\mathrm{CH}_{2}\right), 19.5\left(\mathrm{CH}_{2}\right), 19.5\left(\mathrm{CH}_{3}\right), 14.2\left(\mathrm{CH}_{3}\right)$.

HRMS (ESI): $\mathrm{m} / z$ calcd for $\left[\mathrm{C}_{15} \mathrm{H}_{25} \mathrm{NO}_{2}+\mathrm{H}\right]^{+}:$252.1958; found: 252.1959 . 
Ethyl 1-Benzyl-3-methyloctahydro-1H-cyclopenta[b]pyridine-4acarboxylate (5c)

Prepared according to the general procedure using $6 \mathbf{6 a}(200 \mathrm{mg}, 185$ $\mu \mathrm{L}, 1.28 \mathrm{mmol}, 1.0$ equiv), methacrolein $(\mathbf{8 a} ; 108 \mathrm{mg}, 127 \mu \mathrm{L}, 1.54$ mmol, 1.2 equiv), and benzylamine (7f; $137 \mathrm{mg}, 140 \mu \mathrm{L}, 1.28 \mathrm{mmol}$, 1.0 equiv). The reduction was carried out with $\mathrm{NaBH}_{4}(265 \mathrm{mg})$ in THF $(5 \mathrm{~mL}), \mathrm{AcOH}(3.4 \mathrm{~mL})$, and $\mathrm{EtOH}(1.7 \mathrm{~mL})$. The crude product was obtained with a dr of 10:1. Purification by flash chromatography (PEEtOAc, 1:3) afforded 5c as a yellow oil; yield: $301 \mathrm{mg}(0.998 \mathrm{mmol}$, 78\%); $R_{f}=0.14$ (PE-EtOAc, 1:3; detection: $\mathrm{KMnO}_{4}$ ).

${ }^{1} \mathrm{H} \mathrm{NMR}\left(300 \mathrm{MHz}, \mathrm{CDCl}_{3}\right): \delta=7.45-7.36(\mathrm{~m}, 1 \mathrm{H}), 7.35-7.13(\mathrm{~m}, 4 \mathrm{H})$, $4.10(\mathrm{q}, J=7.1 \mathrm{~Hz}, 2 \mathrm{H}), 4.04(\mathrm{~d}, J=13.5 \mathrm{~Hz}, 1 \mathrm{H}), 3.07(\mathrm{~d}, J=13.5 \mathrm{~Hz}, 1$ H), $2.60(\mathrm{~d}, J=11.0 \mathrm{~Hz}, 1 \mathrm{H}), 2.46(\mathrm{~d}, J=13.1 \mathrm{~Hz}, 1 \mathrm{H}), 2.37-2.21(\mathrm{~m}, 1$ H), 2.08 (dd, $J=12.0,7.9 \mathrm{~Hz}, 1 \mathrm{H}), 1.98-1.79(\mathrm{~m}, 4 \mathrm{H}), 1.73-1.59(\mathrm{~m}, 2$ H), $1.54-1.43(\mathrm{~m}, 1 \mathrm{H}), 1.36(\mathrm{dd}, J=13.1,5.3 \mathrm{~Hz}, 1 \mathrm{H}), 1.28(\mathrm{~d}, J=7.1$ $\mathrm{Hz}, 3 \mathrm{H}), 0.92(\mathrm{~d}, J=7.3 \mathrm{~Hz}, 3 \mathrm{H})$.

${ }^{13} \mathrm{C}$ NMR $\left(75 \mathrm{MHz}, \mathrm{CDCl}_{3}\right): \delta=176.0(\mathrm{C}), 140.4(\mathrm{C}), 128.8(2 \mathrm{CH}), 128.1$ $(2 \mathrm{CH}), 126.6(\mathrm{CH}), 73.6(\mathrm{CH}), 61.0\left(\mathrm{CH}_{2}\right), 60.0\left(\mathrm{CH}_{2}\right), 59.7\left(\mathrm{CH}_{2}\right), 51.2$ (C), $40.6\left(\mathrm{CH}_{2}\right), 37.3\left(\mathrm{CH}_{2}\right), 29.5\left(\mathrm{CH}_{2}\right), 26.4\left(\mathrm{CH}_{2}\right), 19.7(\mathrm{CH}), 19.3$ $\left(\mathrm{CH}_{3}\right), 14.3\left(\mathrm{CH}_{3}\right)$.

HRMS (ESI): $m / z$ calcd for $\left[\mathrm{C}_{19} \mathrm{H}_{27} \mathrm{NO}_{2}+\mathrm{H}\right]^{+}: 302.2114$; found: 302.2115 .

Ethyl 1-(3,4-Dimethoxyphenethyl)-3-methyloctahydro-1H-cyclopenta[b]pyridine-4a-carboxylate (5d)

Prepared according to the general procedure using $6 \mathbf{6}(200 \mathrm{mg}, 185$ $\mu \mathrm{L}, 1.28 \mathrm{mmol}, 1.0$ equiv), methacrolein (8a; $108 \mathrm{mg}, 127 \mu \mathrm{L}, 1.54$ mmol, 1.2 equiv), and 3,4-dimethoxyphenethylamine (7g; $232 \mathrm{mg}$, $217 \mu \mathrm{L}, 1.28 \mathrm{mmol}, 1.0$ equiv). The reduction was carried out with $\mathrm{NaBH}_{4}(325 \mathrm{mg})$ in THF $(9 \mathrm{~mL})$, AcOH $(6 \mathrm{~mL})$, and EtOH $(3 \mathrm{~mL})$. The crude product was obtained with a dr of 10:1. Purification by flash chromatography (PE-EtOAc, 1:3) afforded $\mathbf{5 d}$ as a yellow oil; yield: $427 \mathrm{mg}$ (1.14 mmol, 89\%); $R_{f}=0.10$ (PE-EtOAc, 1:3; detection: UV, vanillin).

${ }^{1} \mathrm{H} \mathrm{NMR}\left(400 \mathrm{MHz}, \mathrm{CDCl}_{3}\right): \delta=6.79-6.72(\mathrm{~m}, 3 \mathrm{H}), 4.07(\mathrm{q}, J=7.1 \mathrm{~Hz}, 2$ H), $3.85(\mathrm{~d}, J=10.6 \mathrm{~Hz}, 6 \mathrm{H}), 2.94$ (ddd, $J=12.4,10.1,6.2 \mathrm{~Hz}, 1 \mathrm{H}$ ), 2.85 $(\mathrm{d}, J=11.0 \mathrm{~Hz}, 1 \mathrm{H}), 2.75-2.69(\mathrm{~m}, 2 \mathrm{H}), 2.44$ (ddd, $J=11.8,9.8,5.6 \mathrm{~Hz}$, $2 \mathrm{H}), 2.36-2.24(\mathrm{~m}, 1 \mathrm{H}), 2.17$ (dd, $J=11.0,3.8 \mathrm{~Hz}, 1 \mathrm{H}), 2.04-1.93(\mathrm{~m}$, 2 H), 1.90-1.79 (m, 2 H), 1.64 (ddd, $J=11.1,9.1,4.1 \mathrm{~Hz}, 2 \mathrm{H}), 1.49-$ $1.40(\mathrm{~m}, 1 \mathrm{H}), 1.37-1.30(\mathrm{~m}, 1 \mathrm{H}), 1.23(\mathrm{t}, J=7.1 \mathrm{~Hz}, 3 \mathrm{H}), 0.97(\mathrm{~d}, J=$ $7.4 \mathrm{~Hz}, 3 \mathrm{H})$.

${ }^{13} \mathrm{C} \operatorname{NMR}\left(75 \mathrm{MHz}, \mathrm{CDCl}_{3}\right): \delta=175.7$ (C), 148.8 (C), $147.2(\mathrm{C}), 134.0$ (C), $120.6(\mathrm{CH}), 112.4(\mathrm{CH}), 111.3(\mathrm{CH}), 73.2(\mathrm{CH}), 60.6\left(\mathrm{CH}_{2}\right), 59.6\left(\mathrm{CH}_{2}\right)$, $57.7\left(\mathrm{CH}_{2}\right), 56.0\left(\mathrm{CH}_{3}\right), 55.9\left(\mathrm{CH}_{3}\right), 50.8(\mathrm{C}), 40.3\left(\mathrm{CH}_{2}\right), 37.1\left(\mathrm{CH}_{2}\right), 32.1$ $\left(\mathrm{CH}_{2}\right), 29.5(\mathrm{CH}), 26.1\left(\mathrm{CH}_{2}\right), 19.6\left(\mathrm{CH}_{3}\right), 19.5\left(\mathrm{CH}_{2}\right), 14.2\left(\mathrm{CH}_{3}\right)$.

HRMS (ESI): $m / z$ calcd for $\left[\mathrm{C}_{22} \mathrm{H}_{33} \mathrm{NO}_{4}+\mathrm{H}\right]^{+}: 376.2482$; found: 376.2480 .

\section{Ethyl 1-[2-(1H-Indol-3-yl)ethyl]-3-methyloctahydro-1H-cyclopen-} ta[b]pyridine-4a-carboxylate (5e)

Prepared according to the general procedure using $\mathbf{6 a}(200 \mathrm{mg}, 185$ $\mu \mathrm{L}, 1.28 \mathrm{mmol}, 1.0$ equiv), methacrolein $(\mathbf{8 a} ; 108 \mathrm{mg}, 127 \mu \mathrm{L}, 1.54$ mmol, 1.2 equiv), and tryptamine ( $7 \mathbf{h} ; 205 \mathrm{mg}, 1.28 \mathrm{mmol}, 1.0$ equiv). The reduction was carried out with $\mathrm{NaBH}_{4}(258 \mathrm{mg})$ in THF $(8 \mathrm{~mL})$, $\mathrm{AcOH}(4 \mathrm{~mL})$, and $\mathrm{EtOH}(2 \mathrm{~mL})$. The crude product was obtained with a dr of 5:1. Purification by flash chromatography (PE-EtOAc 1:3) afforded $\mathbf{5 e}$ as a yellow oil, yield: $363 \mathrm{mg}(1.02 \mathrm{mmol}, 80 \%) ; R_{f}=0.24$ (PE-EtOAc, 1:3; detection: UV, vanillin).
${ }^{1} \mathrm{H} \mathrm{NMR}\left(300 \mathrm{MHz}, \mathrm{CDCl}_{3}\right): \delta=8.05(\mathrm{~s}, 1 \mathrm{H}), 7.61(\mathrm{~d}, J=7.8 \mathrm{~Hz}, 1 \mathrm{H})$ $7.35(\mathrm{~d}, J=7.9 \mathrm{~Hz}, 1 \mathrm{H}), 7.24-7.07(\mathrm{~m}, 3 \mathrm{H}), 4.12(\mathrm{q}, J=7.1 \mathrm{~Hz}, 2 \mathrm{H})$, $3.13(\mathrm{ddd}, J=12.0,9.8,6.6 \mathrm{~Hz}, 1 \mathrm{H}), 2.94(\mathrm{dd}, J=14.5,7.5 \mathrm{~Hz}, 3 \mathrm{H}), 2.59$ (ddd, $J=12.4,9.0,5.1 \mathrm{~Hz}, 1 \mathrm{H}), 2.49$ (d, $J=13.1 \mathrm{~Hz}, 1 \mathrm{H}), 2.36$ (ddd, $J=$ $19.4,11.2,8.8 \mathrm{~Hz}, 1 \mathrm{H}), 2.25-2.17(\mathrm{~m}, 1 \mathrm{H}), 2.13-1.79(\mathrm{~m}, 4 \mathrm{H}), 1.75-$ $1.61(\mathrm{~m}, 2 \mathrm{H}), 1.51(\mathrm{dd}, J=12.3,9.2 \mathrm{~Hz}, 1 \mathrm{H}), 1.40(\mathrm{dd}, J=13.1,5.4 \mathrm{~Hz}$ $1 \mathrm{H}), 1.27(\mathrm{t}, J=7.2 \mathrm{~Hz}, 3 \mathrm{H}), 1.01(\mathrm{~d}, J=7.4 \mathrm{~Hz}, 3 \mathrm{H})$.

${ }^{13} \mathrm{C}$ NMR $\left(75 \mathrm{MHz}, \mathrm{CDCl}_{3}\right): \delta=176.0$ (C), $136.3(\mathrm{C}), 127.9$ (C), 122.2 $(\mathrm{CH}), 121.9(\mathrm{CH}), 119.0(\mathrm{CH}), 118.9(\mathrm{CH}), 115.1(\mathrm{C}), 111.1(\mathrm{CH}), 73.3$ (CH), $60.3\left(\mathrm{CH}_{2}\right), 59.7\left(\mathrm{CH}_{2}\right), 56.0\left(\mathrm{CH}_{2}\right), 50.9(\mathrm{C}), 40.4\left(\mathrm{CH}_{2}\right), 37.2$ $\left(\mathrm{CH}_{2}\right), 29.6(\mathrm{CH}), 26.2\left(\mathrm{CH}_{2}\right), 21.6\left(\mathrm{CH}_{2}\right), 19.6\left(\mathrm{CH}_{2}\right), 19.6\left(\mathrm{CH}_{3}\right), 14.2$ $\left(\mathrm{CH}_{3}\right)$.

HRMS (ESI): $m / z$ calcd for $\left[\mathrm{C}_{22} \mathrm{H}_{30} \mathrm{~N}_{2} \mathrm{O}_{2}+\mathrm{H}\right]^{+}: 355.2380$; found: 355.2378 .

Ethyl 1-Allyl-3-ethyloctahydro-1H-cyclopenta[b]pyridine-4a-carboxylate (5f)

Prepared according to the general procedure using 6a $(200 \mathrm{mg}$, 185 $\mu \mathrm{L}, 1.28 \mathrm{mmol}, 1.0$ equiv), 2-ethylacrolein (8f; $142 \mathrm{mg}, 150 \mu \mathrm{L}, 1.54$ mmol, 1.2 equiv), and allylamine (7e; $73.1 \mathrm{mg}, 95.8 \mu \mathrm{L}, 1.28 \mathrm{mmol}$ 1.0 equiv). The reduction was carried out with $\mathrm{NaBH}_{4}(258 \mathrm{mg})$ in THF $(4 \mathrm{~mL}), \mathrm{AcOH}(2.5 \mathrm{~mL})$, and EtOH $(1.3 \mathrm{~mL})$. The crude product was obtained with a dr of 10:1. Purification by flash chromatography (PEEtOAc, 1:5) afforded $\mathbf{5 f}$ as a yellow oil; yield: $217 \mathrm{mg}(0.819 \mathrm{mmol}$, $64 \%$ ); $R_{f}=0.05$ (PE-EtOAc, 1:5; detection: $\mathrm{KMnO}_{4}$ ).

${ }^{1} \mathrm{H}$ NMR $\left(400 \mathrm{MHz}, \mathrm{CDCl}_{3}\right): \delta=5.94(\mathrm{dddd}, J=17.6,10.2,7.6,5.3 \mathrm{~Hz}, 1$ H), 5.17 (ddd, $J=17.2,3.0,1.9 \mathrm{~Hz}, 1 \mathrm{H}$ ), 5.10 (ddd, $J=10.2,2.8,1.4 \mathrm{~Hz}$, $1 \mathrm{H}), 4.16-3.99(\mathrm{~m}, 2 \mathrm{H}), 3.40$ (ddt, $J=13.9,5.3,1.6 \mathrm{~Hz}, 1 \mathrm{H}$ ), 2.88 (ddd, $J=13.6,6.6,4.8 \mathrm{~Hz}, 2 \mathrm{H}), 2.54(\mathrm{~d}, J=13.3 \mathrm{~Hz}, 1 \mathrm{H}), 2.31-2.20(\mathrm{~m}, 1 \mathrm{H})$, $2.04-1.94(\mathrm{~m}, 2 \mathrm{H}), 1.92-1.85(\mathrm{~m}, 1 \mathrm{H}), 1.81(\mathrm{ddd}, J=16.0,9.2,5.1 \mathrm{~Hz}$ $1 \mathrm{H}), 1.71-1.57(\mathrm{~m}, 3 \mathrm{H}), 1.57-1.50(\mathrm{~m}, 1 \mathrm{H}), 1.48-1.34(\mathrm{~m}, 2 \mathrm{H}), 1.31-$ $1.27(\mathrm{~m}, 1 \mathrm{H}), 1.24(\mathrm{t}, J=7.1 \mathrm{~Hz}, 3 \mathrm{H}), 0.85(\mathrm{t}, J=7.3 \mathrm{~Hz}, 3 \mathrm{H})$.

${ }^{13} \mathrm{C} \mathrm{NMR}\left(101 \mathrm{MHz}, \mathrm{CDCl}_{3}\right): \delta=175.7(\mathrm{C}), 135.7(\mathrm{CH}), 117.2\left(\mathrm{CH}_{2}\right), 73.1$ (CH), $59.7\left(\mathrm{CH}_{2}\right), 59.0\left(\mathrm{CH}_{2}\right), 58.3\left(\mathrm{CH}_{2}\right), 51.1(\mathrm{C}), 38.3\left(\mathrm{CH}_{2}\right), 37.4(\mathrm{CH})$, 37.2 $\left(\mathrm{CH}_{2}\right), 26.0\left(\mathrm{CH}_{2}\right), 25.6\left(\mathrm{CH}_{2}\right), 19.5\left(\mathrm{CH}_{2}\right), 14.2\left(\mathrm{CH}_{3}\right), 13.1\left(\mathrm{CH}_{3}\right)$.

HRMS (ESI): $m / z$ calcd for $\left[\mathrm{C}_{16} \mathrm{H}_{27} \mathrm{NO}_{2}+\mathrm{H}\right]^{+}:$266.2115; found: 266.2114 .

\section{Ethyl 1-Allyl-3-benzyloctahydro-1H-cyclopenta[b]pyridine-4a- carboxylate $(5 \mathrm{~g})$}

Prepared according to the general procedure using $6 \mathbf{6 a}(200 \mathrm{mg}, 185$ $\mu \mathrm{L}, 1.28 \mathrm{mmol}, 1.0$ equiv), 2-benzylacrylaldehyde ( $8 \mathrm{~g} ; 225 \mathrm{mg}, 1.54$ mmol, 1.2 equiv), and allylamine (7e; $73.1 \mathrm{mg}, 95.8 \mu \mathrm{L}, 1.28 \mathrm{mmol}$, 1.0 equiv). The reduction was carried out with $\mathrm{NaBH}_{4}(258 \mathrm{mg})$ in THF $(4 \mathrm{~mL}), \mathrm{AcOH}(2.5 \mathrm{~mL})$, and $\mathrm{EtOH}(1.3 \mathrm{~mL})$. The crude product was obtained with a dr of 10:1. Purification by flash chromatography (PE$\left.\mathrm{Et}_{2} \mathrm{O}, 1: 4\right)$ afforded $\mathbf{5 g}$ as a yellow oil; yield: $268 \mathrm{mg}(0.819 \mathrm{mmol}$, $64 \%) ; R_{f}=0.05$ ( $\mathrm{PE}-\mathrm{Et}_{2} \mathrm{O}, 1: 4$; detection: $\mathrm{KMnO}_{4}$ ).

${ }^{1} \mathrm{H} \mathrm{NMR}\left(400 \mathrm{MHz}, \mathrm{CDCl}_{3}\right): \delta=7.38-7.33(\mathrm{~m}, 2 \mathrm{H}), 7.29-7.23(\mathrm{~m}, 3 \mathrm{H})$, 6.04 (dddd, $J=17.3,10.2,7.9,5.2 \mathrm{~Hz}, 1 \mathrm{H}$ ), 5.25-5.13 (m, $2 \mathrm{H}$ ), 4.32$4.13(\mathrm{~m}, 2 \mathrm{H}), 3.53(\mathrm{ddt}, J=13.7,5.1,1.6 \mathrm{~Hz}, 1 \mathrm{H}), 2.93-2.87(\mathrm{~m}, 2 \mathrm{H})$, 2.84-2.72 (m, $2 \mathrm{H}), 2.64(\mathrm{dd}, J=13.2,4.0 \mathrm{~Hz}, 1 \mathrm{H}), 2.41$ (tdd, $J=11.2$, $10.2,6.5 \mathrm{~Hz}, 1 \mathrm{H}), 2.16-2.09(\mathrm{~m}, 1 \mathrm{H}), 2.04$ (ddd, $J=12.7,9.2,3.8 \mathrm{~Hz}, 2$ H), $2.00-1.88(\mathrm{~m}, 2 \mathrm{H}), 1.83-1.72(\mathrm{~m}, 2 \mathrm{H}), 1.62-1.51(\mathrm{~m}, 2 \mathrm{H}), 1.35(\mathrm{t}$, $J=7.1 \mathrm{~Hz}, 3 \mathrm{H})$.

${ }^{13} \mathrm{C}$ NMR $\left(75 \mathrm{MHz}, \mathrm{CDCl}_{3}\right): \delta=175.6(\mathrm{C}), 142.4(\mathrm{C}), 135.8(\mathrm{CH}), 129.3$ $(2 \mathrm{CH}), 128.2(2 \mathrm{CH}), 125.8(\mathrm{CH}), 117.2\left(\mathrm{CH}_{2}\right), 73.3(\mathrm{CH}), 59.8\left(\mathrm{CH}_{2}\right)$, 59.0 $\left(\mathrm{CH}_{2}\right), 56.7\left(\mathrm{CH}_{2}\right), 51.2(\mathrm{C}), 39.9\left(\mathrm{CH}_{2}\right), 38.9\left(\mathrm{CH}_{2}\right), 37.6(\mathrm{CH}), 37.2$ $\left(\mathrm{CH}_{2}\right), 26.0\left(\mathrm{CH}_{2}\right), 19.6\left(\mathrm{CH}_{2}\right), 14.2\left(\mathrm{CH}_{3}\right)$. 
HRMS (ESI): $\mathrm{m} / z$ calcd for $\left[\mathrm{C}_{21} \mathrm{H}_{29} \mathrm{NO}_{2}+\mathrm{H}\right]^{+}$: 328.2271; found: 328.2268 .

\section{Ethyl 1-Allyl-3-methyldecahydroquinoline-4a-carboxylate (5h)}

Prepared according to the general procedure using ethyl 2-oxocyclohexanecarboxylate (6h; $200 \mathrm{mg}, 187 \mu \mathrm{L}, 1.17 \mathrm{mmol}, 1.0$ equiv), methacrolein (8a; $98.8 \mathrm{mg}, 116 \mu \mathrm{L}, 1.41 \mathrm{mmol}, 1.2$ equiv), and allylamine (7e; $66.8 \mathrm{mg}, 87.5 \mu \mathrm{L}, 1.17 \mathrm{mmol}, 1.0$ equiv). The reduction was carried out with $\mathrm{NaBH}_{4}(240 \mathrm{mg})$ in THF $(5 \mathrm{~mL}), \mathrm{AcOH}(2.7 \mathrm{~mL})$, and EtOH $(1.35 \mathrm{~mL})$. The crude product was obtained with a dr of 6:1. Purification by flash chromatography ( $\left.\mathrm{PE}-\mathrm{Et}_{2} \mathrm{O}, 1: 1\right)$ afforded $\mathbf{5 h}$ as a yellow oil; yield: $276 \mathrm{mg}$ (1.04 mmol, 89\%); $R_{f}=0.05\left(\mathrm{PE}-\mathrm{Et}_{2} \mathrm{O}, 1: 1\right.$; detection: $\left.\mathrm{KMnO}_{4}\right)$.

${ }^{1} \mathrm{H} \mathrm{NMR}\left(400 \mathrm{MHz}, \mathrm{CDCl}_{3}\right): \delta=5.96-5.84(\mathrm{~m}, 1 \mathrm{H}), 5.17-5.07(\mathrm{~m}, 2 \mathrm{H})$ $4.15-4.05(\mathrm{~m}, 2 \mathrm{H}), 3.34$ (dd, $J=14.5,5.9 \mathrm{~Hz}, 1 \mathrm{H}), 3.19(\mathrm{dd}, J=14.5$, $7.0 \mathrm{~Hz}, 1 \mathrm{H}), 2.66(\mathrm{dt}, J=11.3,1.8 \mathrm{~Hz}, 1 \mathrm{H}), 2.38(\mathrm{dd}, J=11.3,3.7 \mathrm{~Hz}, 1$ H), 2.33-2.21 (m, $1 \mathrm{H}), 2.18(\mathrm{dt}, J=13.5,1.9 \mathrm{~Hz}, 1 \mathrm{H}), 1.97(\mathrm{dd}, J=12.1$, $4.0 \mathrm{~Hz}, 1 \mathrm{H}), 1.92-1.78(\mathrm{~m}, 4 \mathrm{H}), 1.45(\mathrm{dd}, J=5.9,3.0 \mathrm{~Hz}, 1 \mathrm{H}), 1.34(\mathrm{dd}$, $J=13.5,5.3 \mathrm{~Hz}, 1 \mathrm{H}), 1.25(\mathrm{t}, J=7.1 \mathrm{~Hz}, 3 \mathrm{H}), 1.28-1.21(\mathrm{~m}, 1 \mathrm{H}), 1.16-$ $1.09(\mathrm{~m}, 2 \mathrm{H}), 0.98(\mathrm{~d}, J=7.3 \mathrm{~Hz}, 3 \mathrm{H})$.

${ }^{13} \mathrm{C} \operatorname{NMR}\left(101 \mathrm{MHz}, \mathrm{CDCl}_{3}\right): \delta=175.8(\mathrm{C}), 135.5(\mathrm{CH}), 117.0\left(\mathrm{CH}_{2}\right), 68.9$ (CH), $60.3\left(\mathrm{CH}_{2}\right), 59.6\left(\mathrm{CH}_{2}\right), 56.0\left(\mathrm{CH}_{2}\right), 46.0(\mathrm{C}), 42.9\left(\mathrm{CH}_{2}\right), 38.7$ $\left(\mathrm{CH}_{2}\right), 29.1(\mathrm{CH}), 26.1\left(\mathrm{CH}_{2}\right), 25.0\left(\mathrm{CH}_{2}\right), 22.1\left(\mathrm{CH}_{2}\right), 19.1\left(\mathrm{CH}_{3}\right), 14.3$ $\left(\mathrm{CH}_{3}\right)$.

HRMS (ESI): $m / z$ calcd for $\left[\mathrm{C}_{16} \mathrm{H}_{27} \mathrm{NO}_{2}+\mathrm{H}\right]^{+}: 266.2115$; found: 266.2116.

\section{Ethyl 1-[2-(1H-indol-3-yl)ethyl]-3-methyldecahydroquinoline-4a- carboxylate (5i)}

Prepared according to the general procedure using $\mathbf{6 h}(200 \mathrm{mg}, 187$ $\mu \mathrm{L}, 1.17 \mathrm{mmol}, 1.0$ equiv), methacrolein (8a; $98.8 \mathrm{mg}, 116 \mu \mathrm{L}, 1.41$ mmol, 1.2 equiv), and tryptamine ( $7 \mathbf{h} ; 188 \mathrm{mg}, 1.17 \mathrm{mmol}, 1.0$ equiv) The reduction was carried out with $\mathrm{NaBH}_{4}(228 \mathrm{mg})$ in THF $(5 \mathrm{~mL})$, AcOH $(3 \mathrm{~mL})$, and EtOH $(1.5 \mathrm{~mL})$. The crude product was obtained with a dr of 6:1. Purification by flash chromatography (PE-EtOAc, $1: 3)$ afforded $\mathbf{5 i}$ as a yellow oil; yield: $310 \mathrm{mg}(0.842 \mathrm{mmol}, 72 \%) ; R_{f}=$ 0.05 (PE-EtOAc, 1:3; detection: $\mathrm{KMnO}_{4}$ ).

${ }^{1} \mathrm{H}$ NMR $\left(400 \mathrm{MHz}, \mathrm{CDCl}_{3}\right): \delta=8.02(\mathrm{~s}, 1 \mathrm{H}), 7.60(\mathrm{~d}, J=7.9 \mathrm{~Hz}, 1 \mathrm{H})$, $7.35(\mathrm{~d}, J=8.0 \mathrm{~Hz}, 1 \mathrm{H}), 7.20-7.15(\mathrm{~m}, 1 \mathrm{H}), 7.13-7.07$ (m, $2 \mathrm{H}), 4.18$ $4.08(\mathrm{~m}, 2 \mathrm{H}), 3.16-3.06(\mathrm{~m}, 1 \mathrm{H}), 3.02-2.89(\mathrm{~m}, 2 \mathrm{H}), 2.87-2.75(\mathrm{~m}, 2$ H), 2.65 (dd, $J=11.1,3.6 \mathrm{~Hz}, 1 \mathrm{H}$ ), 2.37 (ddd, $J=25.2,12.6,4.3 \mathrm{~Hz}, 1 \mathrm{H}$ ) $2.23(\mathrm{~d}, J=13.5 \mathrm{~Hz}, 1 \mathrm{H}), 2.18-2.10(\mathrm{~m}, 1 \mathrm{H}), 1.98-1.81(\mathrm{~m}, 4 \mathrm{H}), 1.50-$ $1.45(\mathrm{~m}, 1 \mathrm{H}), 1.41(\mathrm{dd}, J=13.5,5.3 \mathrm{~Hz}, 1 \mathrm{H}), 1.27(\mathrm{t}, J=7.1 \mathrm{~Hz}, 3 \mathrm{H})$, $1.20-1.15(\mathrm{~m}, 2 \mathrm{H}), 1.02(\mathrm{~d}, J=7.3 \mathrm{~Hz}, 3 \mathrm{H}), 0.97-0.88(\mathrm{~m}, 1 \mathrm{H})$.

${ }^{13} \mathrm{C}$ NMR $\left(101 \mathrm{MHz}, \mathrm{CDCl}_{3}\right): \delta=176.0(\mathrm{C}), 136.3(\mathrm{C}), 127.9(\mathrm{C}), 121.9$ $(\mathrm{CH}), 121.6(\mathrm{CH}), 119.2(\mathrm{CH}), 118.9(\mathrm{CH}), 115.1(\mathrm{C}), 111.2(\mathrm{CH}), 68.7$ $(\mathrm{CH}), 60.0\left(\mathrm{CH}_{2}\right), 59.6\left(\mathrm{CH}_{2}\right), 53.0\left(\mathrm{CH}_{2}\right), 46.1(\mathrm{C}), 43.0\left(\mathrm{CH}_{2}\right), 38.8$ $\left(\mathrm{CH}_{2}\right), 29.1(\mathrm{CH}), 26.2\left(\mathrm{CH}_{2}\right), 25.2\left(\mathrm{CH}_{2}\right), 22.2\left(\mathrm{CH}_{2}\right), 19.8\left(\mathrm{CH}_{2}\right), 19.2$ $\left(\mathrm{CH}_{3}\right), 14.3\left(\mathrm{CH}_{3}\right)$.

HRMS (ESI): $m / z$ calcd for $\left[\mathrm{C}_{23} \mathrm{H}_{32} \mathrm{~N}_{2} \mathrm{O}_{2}+\mathrm{H}\right]^{+}: 369.2537$; found: 369.2536 .

\section{Enantioenriched Ethyl 1-Allyl-3-methyloctahydro-1H-cyclopen-} ta[b]pyridine-4a-carboxylate $(5 b)$

A $25 \mathrm{~mL}$ flask filled with argon was equipped with a magnetic stirre bar. Toluene $(5 \mathrm{~mL})$ was then added followed by organocatalyst III (12.3 mg, $0.032 \mathrm{mmol}, 10 \mathrm{~mol} \%$ ). Then, ethyl 2-oxocyclopentanecarboxylate (6a; $50 \mathrm{mg}, 46.3 \mu \mathrm{L}, 0.32 \mathrm{mmol}, 1.0$ equiv) was added followed by methacrolein (8a; $31.2 \mu \mathrm{L}, 0.38 \mathrm{mmol}, 1.2$ equiv), and allylamine (7e; $24.0 \mu \mathrm{L}, 0.32 \mathrm{mmol}, 1.0$ equiv). After complete conversion of the $\beta$-keto ester, checked by TLC (eluent: PE-EtOAc, 1:3), the solution was filtered through a short pad of Celite, which was thoroughly washed with toluene. The solvent was evaporated under reduced pressure to obtain the crude dieneamine. In a $50 \mathrm{~mL}$ flask equipped with a magnetic stirring bar, were added the crude dieneamine dissolved in $\mathrm{THF}(2 \mathrm{~mL})$, and $\mathrm{NaBH}_{4}$ ( $72.6 \mathrm{mg}, 1.92 \mathrm{mmol}, 6$ equiv) under argon. Then, AcOH ( $1 \mathrm{~mL})$ was slowly added (caution: gas evolution), followed by EtOH $(0.5 \mathrm{~mL})$. The resultant suspension was stirred at r.t. for $24 \mathrm{~h}$. After completion of the reaction, most of the solvent was removed under reduced pressure. $\mathrm{H}_{2} \mathrm{O}(5 \mathrm{~mL}), 10 \%$ aq $\mathrm{NaOH}(5 \mathrm{~mL})$, and $\mathrm{Et}_{2} \mathrm{O}(5 \mathrm{~mL})$ were added. The organic layer was separated and the aqueous phase was further extracted with $\mathrm{Et}_{2} \mathrm{O}(3 \times 10 \mathrm{~mL})$. The combined organic layers were then washed with $\mathrm{H}_{2} \mathrm{O}(2 \times 10 \mathrm{~mL})$, brine $(15 \mathrm{~mL})$, and dried $\left(\mathrm{Na}_{2} \mathrm{SO}_{4}\right)$. The solvent was evaporated giving the crude product, which was analyzed by ${ }^{13} \mathrm{C}$ NMR spectroscopy to measure the diastereomeric ratio. The crude product was obtained with a $\mathrm{dr}$ of $10: 1$. Purification was performed on a silica gel column affording the pure product $\mathbf{5 b}$ ( $65 \%$ yield, $45 \%$ ee). Analyses were in accordance with those obtained when the reaction was performed with $4 \AA$ MS Analyses were identical to the ones obtained for the racemic product. HPLC: $(S, S)$-Whelk-O1, hexane-i-PrOH $+\mathrm{Et}_{3} \mathrm{~N}(98: 2), 25^{\circ} \mathrm{C}, 0.5$ $\mathrm{mL} / \mathrm{min}, \lambda=230 \mathrm{~nm}, t_{\mathrm{R}}($ major $)=8.28 \mathrm{~min}, t_{\mathrm{R}}(\operatorname{minor})=9.23 \mathrm{~min}$.

\section{Ethyl 4-(Allylamino)-3-methyl-8-oxobicyclo[3.2.1 ]octane-1-car-} boxylate (9)

A $25 \mathrm{~mL}$ two-necked flask filled with argon was equipped a magnetic stirrer bar. Toluene $(5 \mathrm{~mL})$ was then added followed by organocatalyst V (13.2 mg, $0.032 \mathrm{mmol}, 10 \mathrm{~mol} \%)$. Then, ethyl 2-oxocyclopentanecarboxylate (6a; $50 \mathrm{mg}, 46.3 \mu \mathrm{L}, 0.32 \mathrm{mmol}, 1.0$ equiv) was added followed by methacrolein $(\mathbf{8 a} ; 31.2 \mu \mathrm{L}, 0.38 \mathrm{mmol}, 1.2$ equiv), and allylamine (7e; $24.0 \mu \mathrm{L}, 0.32 \mathrm{mmol}, 1.0$ equiv). After complete conversion of 6a, checked by TLC (eluent: PE-EtOAc-Et $\left.{ }_{3} \mathrm{~N}, 3: 1: 0.025\right)$, the solution was filtered through a short pad of Celite, which was thoroughly washed with toluene. The solvent was evaporated under reduced pressure to obtain crude compound, which was analyzed by ${ }^{13} \mathrm{C}$ NMR spectroscopy to measure the diastereomeric ratio. The crude product was obtained with a $\mathrm{dr}$ of $6: 1$. Purification was performed on a silica gel column affording the pure product $\mathbf{9}$ ( $60 \%$ yield, $6 \%$ ee). The racemate was prepared following the same procedure using racemic organocatalyst $\mathbf{V} ; R_{f}=0.65\left(\mathrm{PE}-\mathrm{EtOAc}-\mathrm{Et}_{3} \mathrm{~N}, 3: 1: 0.025\right.$; detection: $\mathrm{KMnO}_{4}$ ).

HPLC: Chiralpak AD-H, hexane-EtOH (95:5), $25^{\circ} \mathrm{C}, 1 \mathrm{~mL} / \mathrm{min}, \lambda=220$ $\mathrm{nm}, t_{\mathrm{R}}($ major $)=6.80 \mathrm{~min}, t_{\mathrm{R}}($ minor $)=8.31 \mathrm{~min}$.

${ }^{1} \mathrm{H} \mathrm{NMR}\left(400 \mathrm{MHz}, \mathrm{CDCl}_{3}\right.$ ): $\delta=5.79$ (dddd, $J=16.9,10.2,6.5,5.6 \mathrm{~Hz}, 1$ $\mathrm{H}), 5.11$ (ddd, $J=17.2,3.2,1.6 \mathrm{~Hz}, 1 \mathrm{H}$ ), 5.01 (ddd, $J=10.2,2.9,1.5 \mathrm{~Hz}$ $1 \mathrm{H}), 4.23-4.05(\mathrm{~m}, 2 \mathrm{H}), 3.39(\mathrm{ddt}, J=14.1,5.5,1.5 \mathrm{~Hz}, 1 \mathrm{H}), 3.15-3.05$ $(\mathrm{m}, 1 \mathrm{H}), 2.94$ (td, $J=4.5,2.0 \mathrm{~Hz}, 1 \mathrm{H}), 2.59-2.45(\mathrm{~m}, 2 \mathrm{H}), 2.36-2.22$ $(\mathrm{m}, 1 \mathrm{H}), 2.16(\mathrm{td}, J=13.1,1.3 \mathrm{~Hz}, 1 \mathrm{H}), 2.00$ (dddd, $J=15.1,13.1,9.0$ $4.5 \mathrm{~Hz}, 2 \mathrm{H}), 1.85$ (ddd, $J=13.1,4.9,2.0 \mathrm{~Hz}, 1 \mathrm{H}), 1.74-1.62(\mathrm{~m}, 1 \mathrm{H})$, $1.22(\mathrm{t}, J=7.1 \mathrm{~Hz}, 3 \mathrm{H}), 0.96(\mathrm{~d}, J=6.6 \mathrm{~Hz}, 3 \mathrm{H})$.

${ }^{13} \mathrm{C}$ NMR $\left(101 \mathrm{MHz}, \mathrm{CDCl}_{3}\right): \delta=212.5(\mathrm{C}), 171.3(\mathrm{C}), 137.1(\mathrm{CH}), 116.0$ $\left(\mathrm{CH}_{2}\right), 67.1(\mathrm{CH}), 61.1\left(\mathrm{CH}_{2}\right), 57.0(\mathrm{C}), 50.0\left(\mathrm{CH}_{2}\right), 48.6(\mathrm{CH}), 42.0\left(\mathrm{CH}_{2}\right)$, $28.5(\mathrm{CH}), 27.0\left(\mathrm{CH}_{2}\right), 20.2\left(\mathrm{CH}_{2}\right), 16.3\left(\mathrm{CH}_{3}\right), 14.2\left(\mathrm{CH}_{3}\right)$.

\section{Acknowledgment}

We thank Dr. N. Vanthuyne and M. Jean for HPLC analyses on chiral stationary phase, and the whole team of the Spectropole (www.spectropole.fr). Financial support from Aix Marseille Université, Centrale 
Marseille, the CNRS, the COST action CM0905 ORCA, and the China Scholarship Council (scholarship for H.D.) is acknowledged.

\section{Supporting Information}

Supporting information for this article is available online at http://dx.doi.org/10.1055/s-0034-1380229.

\section{References}

(1) For monographs and reviews on multicomponent reactions, see: (a) Zhu, J.; Bienaymé, H. Multicomponent Reactions; WileyVCH: Weinheim, 2005. (b) Touré, B. B.; Hall, D. G. Chem. Rev. 2009, 109, 4439. (c) Ruijter, E.; Scheffelaar, R.; Orru, R. V. A. Angew. Chem. Int. Ed. 2011, 50, 6234. (d) Climent, M. J.; Corma A.; Iborra, S. RSC Adv. 2012, 2, 16. (e) Dömling, A.; Wang, W.; Wang, K. Chem. Rev. 2012, 112, 3083. (f) Brauch, S.; van Berkel, S. S.; Westermann, B. Chem. Soc. Rev. 2013, 42, 4948. (g) Cioc, R. C.; Ruijter, E.; Orru, R. V. A. Green Chem. 2014, 16, 2958.

(2) For reviews on the application of multicomponent reactions to the synthesis of heterocycles, see: (a) Jiang, B.; Rajale, T.; Wever, W.; Tu, S.-J.; Li, G. Chem. Asian J. 2010, 5, 2318. (b) Bugaut, X.; Bonne, D.; Coquerel, Y.; Rodriguez, J.; Constantieux, T. Curr. Org. Chem. 2013, 17, 1920.

(3) For reviews and book chapters, see: (a) Simon, C.; Constantieux, T.; Rodriguez, J. Eur. J. Org. Chem. 2004, 4957. (b) Liéby-Muller F.; Simon, C.; Constantieux, T.; Rodriguez, J. QSAR Comb. Sci. 2006, 25, 432. (c) Bonne, D.; Coquerel, Y.; Constantieux, T.; Rodriguez, J. Tetrahedron: Asymmetry 2010, 21, 1085 (d) Sanchez Duque, M. M.; Allais, C.; Isambert, N.; Constantieux, T.; Rodriguez, J. Top. Heterocycl. Chem. 2010, 23, 227. (e) Isambert, N.; Sanchez Duque, M. M.; Plaquevent, J.-C.; Génisson, Y.; Rodriguez, J.; Constantieux, T. Chem. Soc. Rev. 2011, 40, 1347. (f) Bonne, D.; Constantieux, T.; Coquerel, Y.; Rodriguez, J. Chem. Eur. J. 2013, 19, 2218. (g) Rodriguez, J. Bonne, D.; Coquerel, Y.; Constantieux, T. In Science of Synthesis Multicomponent Reactions 2; Müller, T. J. J., Ed.; Thieme: Stuttgart, 2014, 1-36.

(4) (a) Simon, C.; Lieby-Muller, F.; Peyronel, J.-F.; Constantieux, T. Rodriguez, J. Synlett 2003, 2301. (b) For preliminary studies using Dowex basic ion-exchange resins as promoters, see: Simon, C.; Peyronel, J.-F.; Clerc, F.; Rodriguez, J. Eur. J. Org. Chem. 2002, 3359. (c) For an early contribution using $\alpha$-unsubstituted 1,3-dicarbonyls and $\alpha, \beta$-unsaturated imines, see: Geirsson, J. K. F.; Johannesdottir, J. F. J. Org. Chem. 1996, 61, 7320.

(5) For reviews on the construction of bicyclo[3.2.1]octanes, see: (a) Presset, M.; Coquerel, Y.; Rodriguez, J. ChemCatChem 2012, 4 172. (b) Presset, M.; Coquerel, Y.; Rodriguez, J. Chem. Rev. 2013 113,525 .

(6) For selected examples, see: (a) Stork, G.; Landesman, H. K. J. Am. Chem. Soc. 1956, 78, 5129. (b) Hendrickson, J. B.; Boeckman, R. K. J. Am. Chem. Soc. 1971, 93, 1307. (c) Murray, D. F.; Baum, M. W.; Jones, M. J. Org. Chem. 1986, 51, 1. (d) Ravishankar, L.; Trivedi, G. K. Indian J. Chem., Sect. B: Org. Chem. Incl. Med. Chem. 1989, 28, 303. (e) Hong, X.; France, S.; Mejía-Oneto, J. M.;
Padwa, A. Org. Lett. 2006, 8, 5141. (f) Hong, X.; France, S.; Padwa, A. Tetrahedron 2007, 63, 5962. (g) Corkey, B. K.; Heller, S. T.; Wang, Y.-M.; Toste, F. D. Tetrahedron 2013, 69, 5640.

(7) (a) Sanchez Duque, M. M.; Baslé, O.; Génisson, Y.; Plaquevent, J.C.; Bugaut, X.; Constantieux, T.; Rodriguez, J. Angew. Chem. Int. Ed. 2013, 52, 14143. (b) Du, H.; Rodriguez, J.; Bugaut, X.; Constantieux, T. Adv. Synth. Catal. 2014, 356, 851.

(8) For contributions of other research groups, see: (a) A pseudo three-component reaction initiated by an organocatalytic enantioselective Michael addition of a 1,3-dicarbonyl compound: Bertelsen, S.; Johansen, R. L.; Jørgensen, K. A. Chem. Commun. 2008, 3016. (b) A three-component reaction involving the addition to an in situ generated $\alpha, \beta$-unsaturated imine: Jiang, J.; Yu J.; Sun, X.-X.; Rao, Q.-Q.; Gong, L.-Z. Angew. Chem. Int. Ed. 2008, 47, 2458. (c) An enantioselective Hantzsch synthesis of polyhydroquinolines: Evans, C. G.; Gestwicki, J. E. Org. Lett. 2009, 11, 2957. (d) A three-component reaction involving a conjugate addition to nitroolefins: Wang, Y.; Han, R.-G.; Zhao, Y.-L.; Yang, S.; Xu, P.-F.; Dixon, D. J. Angew. Chem. Int. Ed. 2009, 48, 9834.

(9) For reviews on organocatalytic enantioselective multicomponent reactions, see: (a) Marson, C. M. Chem. Soc. Rev. 2012, 41 7712. (b) de Graaff, C.; Ruijter, E.; Orru, R. V. A. Chem. Soc. Rev. 2012, 41, 3969. (c) Volla, C. M. R.; Atodiresei, I.; Rueping, M. Chem. Rev. 2014, 114, 2390.

(10) For a review on catalytic selective synthesis, see: Mahatthananchai, J.; Dumas, A. M.; Bode, J. W. Angew. Chem. Int. Ed. 2012, 51, 10954.

(11) Filippini, M.-H.; Faure, R.; Rodriguez, J. J. Org. Chem. 1995, 60, 6872.

(12) Erkkilä, A.; Majander, I.; Pihko, P. M. Chem. Rev. 2007, 107, 5416.

(13) Doyle, A. G.; Jacobsen, E. N. Chem. Rev. 2007, 107, 5713.

(14) (a) Marigo, M.; Wabnitz, T. C.; Fielenbach, D.; Jørgensen, K. A. Angew. Chem. Int. Ed. 2005, 44, 794. (b) Hayashi, Y.; Gotoh, H.; Hayashi, T.; Shoji, M. Angew. Chem. Int. Ed. 2005, 44, 4212. (c) Xu, L.-W.; Li, L.; Shi, Z.-H. Adv. Synth. Catal. 2010, 352, 243. (d) Jensen, K. L.; Dickmeiss, G.; Jiang, H.; Albrecht, Ł.; Jørgensen, K. A. Acc. Chem. Res. 2012, 45, 248. (e) Meninno, S.; Lattanzi, A. Chem. Commun. 2013, 49, 3821.

(15) (a) Wu, L.-Y.; Bencivenni, G.; Mancinelli, M.; Mazzanti, A.; Bartoli, G.; Melchiorre, P. Angew. Chem. Int. Ed. 2009, 48, 7196. (b) Melchiorre, P. Angew. Chem. Int. Ed. 2012, 51, 9748. (c) Cassani, C.; Martín-Rapún, R.; Arceo, E.; Bravo, F.; Melchiorre, P. Nat. Protoc. 2013, 8, 325.

(16) Fuerst, D. E.; Jacobsen, E. N. J. Am. Chem. Soc. 2005, 127, 8964

(17) Yang, W.; Du, D.-M. Adv. Synth. Catal. 2011, 353, 1241.

(18) (a) Okino, T.; Hoashi, Y.; Takemoto, Y. J. Am. Chem. Soc. 2003 125, 12672. (b) Okino, T.; Hoashi, Y.; Furukawa, T.; Xu, X.; Takemoto, Y.J. Am. Chem. Soc. 2005, 127, 119.

(19) (a) Vakulya, B.; Varga, S.; Csámpai, A.; Soós, T. Org. Lett. 2005, 7, 1967. (b) McCooey, S. H.; Connon, S. J. Angew. Chem. Int. Ed. 2005, 44, 6367.

(20) (a) Malerich, J. P.; Hagihara, K.; Rawal, V. H. J. Am. Chem. Soc. 2008, 130, 14416. (b) Alemán, J.; Parra, A.; Jiang, H.; Jørgensen, K. A. Chem. Eur. J. 2011, 17, 6890. (c) Storer, R. I.; Aciro, C.; Jones, L. H. Chem. Soc. Rev. 2011, 40, 2330.

(21) Given the low enantiomeric excesses of products $\mathbf{5 b}$ and $\mathbf{9}$, no attempt was made to determine the absolute configuration of the major enantiomer.

(22) Schmidt, Y.; Breit, B. Chem. Eur. J. 2011, 17, 11780. 